\title{
Laser-assisted XUV few-photon double ionization of helium: Joint angular distributions
}

\author{
Aihua Liu and Uwe Thumm \\ James R. Macdonald Laboratory, Department of Physics, Kansas State University, Manhattan, Kansas 66506-2604, USA
}

(Received 31 March 2014; published 30 June 2014)

\begin{abstract}
We investigate few-photon extreme ultraviolet (XUV) double ionization of helium atoms without and in the presence of an assisting infrared (IR) laser field by numerically solving the time-dependent Schrödinger equation in full dimensionality within a finite-element discrete-variable-representation scheme. We discuss joint energy distributions for coplanar emission where the emitted electron momenta and polarization axis of the linearly polarized XUV and IR pulses lie in a plane. Our analysis focuses on joint angular distributions for highly correlated equal-energy-sharing double ionization by absorption of one, two, or three XUV photons and IR-laser-assisted single-photon XUV double ionization.
\end{abstract}

DOI: 10.1103/PhysRevA.89.063423

PACS number(s): 32.80.Fb, 42.50.Hz, 31.15.vj

\section{INTRODUCTION}

The exact quantitative description and full understanding of the dynamics of three or more interacting particles is a fundamental and ongoing problem in quantum mechanics. While one of the most elementary systems for studying the dynamics of three Coulomb-interacting particles, the helium atom, has been examined extensively during the past few decades, three-particle Coulomb breakup initiated by short and intense pulses of electromagnetic radiation in the infrared (IR) to the extreme ultraviolet (XUV) spectral range continues to be of particular interest to experimentalists and theorists alike. The complete investigation of such three-particle breakup processes remains a demanding experiment that requires advanced laser or synchrotron and coincident particle detection systems [1-6]. In such experiments, the momenta of two particles in the final, fully fragmented state, consisting of the helium nucleus and the two released electrons, need to be detected in coincidence. On the theoretical side, the quantitative description of the three-body breakup process has made significant progress over the past two decades [7-18], most noticeably due to the development of improved analytical models for the correlated final three-body Coulomb state after the three-particle breakup [19,20] and extensive $a b$ initio calculations made possible by efficient numerical algorithms [21,22] and much improved computational resources.

Despite the complicated nature of the three-particle Coulomb-breakup dynamics in response to the absorption of one $[3,4,8,15,20]$, a few $[6,8-16]$, or many $[17,18]$ photons by helium atoms in their ground state, basic physical principles remain transparent in measured differential helium doubleionization (DI) cross sections. These underlying principles include most importantly angular-momentum, energy, and parity conservation, and selection rules for the interaction of atoms with electromagnetic radiation. They constitute a link between the symmetry properties of the bound initial and three-body final continuum state of the ground-state and ionized helium atom, respectively, and help significantly in understanding the comprehensive information contained in both measured and numerically calculated differential DI cross sections as functions of the two emitted electrons' momenta [20]. For example for "coplanar" emission geometry, where the emitted electron momenta and polarization axis of the incident linearly polarized XUV radiation lie in a plane, the DI probability can be expressed as a sum of terms that each consist of a dynamical and an angular factor. While the dynamical factors are typically complicated functions of the kinetic energies $E_{1}$ and $E_{2}$ of the released photoelectrons and their relative emission angle $\theta_{1,2}=\theta_{2}-\theta_{1}$, the angular functions are simple expressions that only depend on the emission angles $\theta_{1}$ and $\theta_{2}$. In particular, for equal energy sharing $\left(E_{1}=E_{2}\right)$, the probability for DI by a single XUV photon factorizes into a single dynamical factor and the angular factor $\left(\cos \theta_{1}+\cos \theta_{2}\right)^{2}$, reminiscent of uncorrelated dipolar angular distributions and implying the absence of "back-to-back emission" [Fig. 1(a)]. For two-photon DI at equal energy sharing and coplanar emission, the DI yield is determined by four dynamical and four angular factors, and thus much less analytically tractable than single-photon DI yields [9].

Early XUV synchrotron radiation experimental results for the triply differential direct double photoionization in helium were reported by Schwarzkopf et al. [1], in excellent agreement with the calculation of Maulbetsch and Briggs [7]. These DI cross sections were obtained for equal energy sharing of the emitted electrons and for coplanar emission geometry. Bräuning et al. [3] measured absolute triply differential cross sections for photo-DI of helium covering the full range of possible energy sharing and emission angles of the two photoelectrons. We note that the references addressed in this work constitute a small and not representative part of the very large number of investigations on the DI of helium atoms and refer the reader to more comprehensive reviews for single-photon [20,23,24] and two-photon [9,11,12,16,24] $\mathrm{DI}$, and references therein.

DI of helium is a convoluted process where photon-electron, electron-nucleus, and electronic interactions compete. Accordingly, the detailed scrutiny of measured and calculated DI data requires highly differential emission probabilities (or cross sections). We therefore focus in this work on a specific value of the energy sharing between the two emitted electrons, equal energy sharing, and investigate highly differential joint angular distributions (JADs) for coplanar emission. We point out that for coplanar emission and equal energy sharing, JADs correspond to triply differential DI cross section (TDCS), both terms, JAD and TDCS, being used alternatively in the literature. We will consider short XUV pulses with photon 


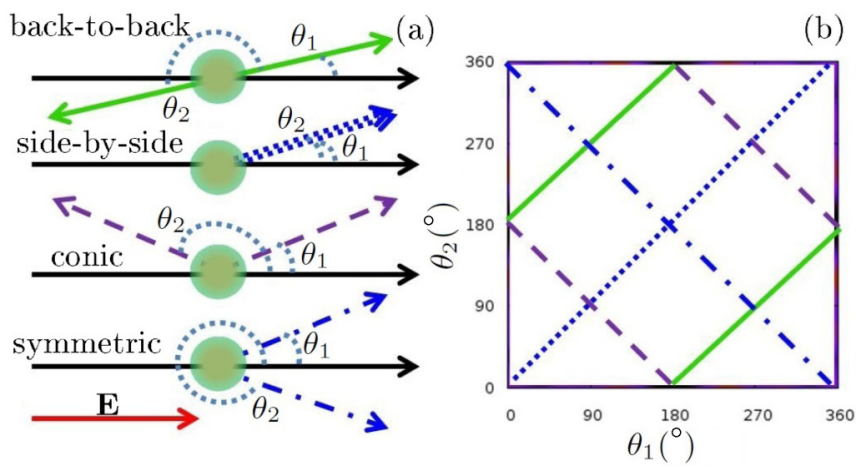

FIG. 1. (Color online) (a) Schematics for four different types of photoelectron emission patterns in the double ionization of helium. From top to bottom: back-to-back emission, side-by-side emission, conic emission, and symmetric emission. (b) Schematics for the identification of the corresponding emission patterns (with matching line types and colors) in joint angular distributions (see text).

energies $\hbar \omega_{\mathrm{XUV}}$ between 30 and $99 \mathrm{eV}$, thus covering the nonsequential and sequential DI regimes.

JADs for helium DI exhibit four different typical electron emission patterns (Fig. 1), which we will refer to repeatedly throughout this paper. For coplanar emission, each pattern can be related to a given range of $\theta_{1}$ and $\theta_{2}$ relative to the linear polarization direction of the XUV and/or IR electric field, which will serve as the quantization $(z)$ axis in our calculation below. The red solid arrow in Fig. 1(a) indicates the XUV or IR electric-field direction $\mathbf{E}$, and the remaining arrows represent photoelectron momenta. Different colors and line styles correspond to different emission patterns in the JADs, as schematically depicted in Fig. 1(b):

(i) "back-to-back emission" [green solid arrows in Fig. 1(a) and green solid lines with slope $45^{\circ}$ in Fig. 1(b) with $\theta_{12}=$ $\left.\left|\theta_{1}-\theta_{2}\right|=180^{\circ}\right]$

(ii) "side-by-side emission" [blue dotted arrows in Fig. 1(a) and blue dotted line in Fig. 1(b) with $\theta_{1}=\theta_{2}$ ];

(iii) "conic emission" [purple dashed arrows in Fig. 1(a) and purple dashed lines with slope $-45^{\circ}$ and $\theta_{1}+\theta_{2}=360^{\circ} \pm$ $180^{\circ}$ ]; and

(iv) "symmetric emission" [blue dotted-dashed arrows in Fig. 1(a) and blue dotted-dashed line along $\theta_{1}+\theta_{2}=360^{\circ}$ in Fig. 1(b)].

We will investigate few-XUV-photon coplanar DI of helium by solving the time-dependent Schrödinger equation (TDSE) within a finite-element discrete-variable-representation (FEDVR) scheme. For this purpose we developed a numerical code. We start by reviewing our theoretical model and key features of its numerical implementation in Sec. II. In Sec. III A we discuss energy- and angle-differential correlated electron-emission probabilities for DI due to the absorption of up to $N_{\mathrm{XUV}}=3$ photons, reproducing measured angular distributions for single-photon DI. In Sec. III B we investigate the DI of helium by absorption of a single XUV photon assisted by an IR laser pulse and discuss energy- and angle-resolved photoemission probabilities in view of the added influence the absorption or emission of (effectively) $N_{\text {IR }}$ IR photons has on the photoemission process. In this discussion, we focus on JADs for specific numbers of effectively absorbed (or emitted) IR photons. These JADs are thus differential not only with regard to the momenta of both emitted electrons, but also in $N_{\text {IR }}$. They provide-to our knowledge-the most highly resolved theoretical double-photoemission data available. As we will show, the assistance of the IR field induces characteristic modifications of the IR-laser-free JADs that depend on $N_{\mathrm{IR}}$. We conclude this work with a summary in Sec. IV. This paper includes two appendixes. Appendix A gives details on our implementation of the angular momentum coupling. Importantly, in order to validate our numerical model, we carefully assess in Appendix B the dependence of our numerical results with regard to their convergence in numerical and physical parameters and compare our groundand excited-state energies for the helium atom with theoretical and experimental energies from the literature. In order to most critically test the convergence properties of our numerical scheme, we examine JADs for physical parameters of the emitted electrons at which electronic correlation matters most, i.e., for equal energy sharing and side-by-side emission. Unless otherwise stated, we use atomic units throughout this paper. Furthermore, unless specified differently, we display joint-energy distributions and JADs at their maximal yields and color (gray-scale)-coded on a linear scale.

\section{THEORY AND NUMERICAL IMPLEMENTATION}

Our theoretical method for calculating energy and angle differential DI processes for helium is based on the $a b$ initio numerical solution of the TDSE for two electrons subject to their mutual interaction and their interactions with the nucleus and the external electric fields of the XUV and IR laser pulses,

$$
i \frac{\partial \Phi\left(\mathbf{r}_{1}, \mathbf{r}_{2} ; t\right)}{\partial t}=H \Phi\left(\mathbf{r}_{1}, \mathbf{r}_{2} ; t\right)
$$

with the Hamiltonian

$$
H=H_{0}+V_{\text {int }},
$$

where

$$
H_{0}=-\frac{1}{2}\left[\nabla_{1}^{2}+\nabla_{2}^{2}\right]-\frac{2}{r_{1}}-\frac{2}{r_{1}} .
$$

The interaction potential

$$
V_{\mathrm{int}}=\frac{1}{\left|\mathbf{r}_{1}-\mathbf{r}_{2}\right|}+\left[\mathbf{E}_{\mathrm{XUV}}(t)+\mathbf{E}_{\mathrm{IR}}(t)\right] \cdot\left(\mathbf{r}_{1}+\mathbf{r}_{2}\right)
$$

includes the electronic correlation and interactions between the electrons and external XUV and IR electric fields in the dipole length gauge. We assume the external XUV and IR electric fields to be linearly polarized along the quantization (z) axis and to have $\cos ^{2}$ temporal profiles,

$$
E_{a}(t)=\left\{\begin{array}{lll}
E_{0, a} \cos ^{2}\left(\frac{\pi}{2} \frac{t}{\tau_{a}}\right) \cos \left(\omega_{a} t+\varphi_{a}\right), & \text { if } & |t|<\tau_{a}, \\
0, & \text { if } & |t|>\tau_{a},
\end{array}\right.
$$

where the index " $a$ " stands for XUV or IR and $E_{0, a}, \tau_{a}$, $\varphi_{a}$, and $\omega_{a}$ denote the electric-field amplitudes, pulse lengths, phases, and frequencies of the two pulses, respectively. We will not discuss the carrier-envelope-phase dependencies of the DI process in this work and set $\varphi_{a}=0$ for both XUV and IR pulses. 
We solve (1) by expanding the time-dependent two-electron wave function in bipolar spherical harmonics,

$$
\mathcal{Y}_{l_{1} l_{2}}^{L M}\left(\Omega_{1}, \Omega_{2}\right)=\sum_{m_{1} m_{2}} C_{l_{1} m_{1} l_{2} m_{2}}^{L M} Y_{l_{1} m_{1}}\left(\Omega_{1}\right) Y_{l_{2} m_{2}}\left(\Omega_{2}\right),
$$

according to

$$
\Phi\left(\mathbf{r}_{1}, \mathbf{r}_{2} ; t\right)=\sum_{L M} \sum_{l_{1}, l_{2}} \frac{\psi_{l_{1} l_{2}}^{(L M)}\left(r_{1}, r_{2} ; t\right)}{r_{1} r_{2}} \mathcal{Y}_{l_{1} l_{2}}^{L, M}\left(\Omega_{1}, \Omega_{2}\right) .
$$

The functions $Y_{l_{i} m_{i}}\left(\Omega_{i}\right)$ are ordinary spherical harmonics in the solid angles $\Omega_{i}=\left(\theta_{i}, \phi_{i}\right)$ of the two electrons $(i=1$, 2 ). The coupling of the two electrons' individual angular momenta with quantum numbers $\left(l_{1}, m_{1}\right)$ and $\left(l_{2}, m_{2}\right)$ to the total angular momentum of the two-electron system with quantum numbers $(L, M)$ is written in terms of the Clebsch-Gordan coefficients $C_{l_{1} m_{1} l_{2} m_{2}}^{L M}$. Assuming the helium atoms are initially in the singlet-spin ${ }^{1} S_{0}$ ground state with $L=M=0$, angular momentum conservation implies that the atoms remain in $M=0$ states during their interaction with the XUV and IR electric fields. Starting with helium atoms in the ${ }^{1} S_{0}$ state further implies that the radial parts of the atomic wave function are symmetric upon electron exchange, i.e., $\Phi\left(\mathbf{r}_{1}, \mathbf{r}_{2}\right)=\Phi\left(\mathbf{r}_{2}, \mathbf{r}_{1}\right)$, such that for our case $(M=0)$ only terms with even $L-l_{1}-l_{2}$ contribute to the expansion (6).

In order to obtain energy and angle-dependent doubleionization probabilities, we need to analyze the final state of the doubly ionized atom with regard to the asymptotic momentum vectors of both emitted electrons. In principle, quantum mechanics demands this to be done by projecting the wave function of the system, $\Phi\left(\mathbf{r}_{1}, \mathbf{r}_{2} ; t_{f}\right)$, at a sufficiently long time $t_{f}$ after the action of the IR and XUV pulses onto asymptotic two-electron continuum wave functions. Since no accurate closed-form expressions of such asymptotic correlated twoelectron wave functions are known for the three-body Coulomb problem [11], we proceed by neglecting the final-state interaction between asymptotic emitted electrons, leaving the inclusion of electronic correlation in the final state [19] to future investigations. This allows us to approximately compute DI probabilities by subtracting the ${ }^{1} S_{0}$ ground-state wave function from $\Phi\left(\mathbf{r}_{1}, \mathbf{r}_{2} ; t_{f}\right)$ before projecting this difference onto products of incoming-wave Coulomb continuum wave functions $\psi_{\mathbf{k}_{i}}^{(-)}\left(\mathbf{r}_{i}\right)(i=1,2)$ with emitted electron momenta $\mathbf{k}_{1}$ and $\mathbf{k}_{2}$,

$$
\begin{aligned}
& P\left(\mathbf{k}_{1}, \mathbf{k}_{2}\right) \\
& \quad=\mid\left\langle\left.\psi_{\mathbf{k}_{1}}^{(-)}\left(\mathbf{r}_{1}\right) \psi_{\mathbf{k}_{2}}^{(-)}\left(\mathbf{r}_{2}\right)\left[\left|\Phi\left(\mathbf{r}_{1}, \mathbf{r}_{2} ; t_{f}\right)\right\rangle-\left|\Phi_{S}\left(\mathbf{r}_{1}, \mathbf{r}_{2}\right)\right\rangle\right]\right|^{2} .\right.
\end{aligned}
$$

We compute the wave functions $\psi_{\mathbf{k}}^{(-)}(\mathbf{r})$ with the routine COULFG of Barnett [25]. As long as ionization by more than two photons is irrelevant, single-photon and two-photon DI probabilities can be separated by restricting the sum in (7) to include either only odd or only even total angular momentum quantum numbers $L$, respectively. In general, starting with the $L=0$ ground state of helium, we obtain DI probabilities for even (odd) numbers of absorbed (minus emitted) photons by restricting the sum in (7) to even (odd) values of $L$.

Based on (8), we evaluate JADs for coplanar DI at vanishing azimuthal angles $\left(\phi_{1}=\phi_{2}=0\right)$ and for fixed equal energy sharing $\left(k_{1}^{2}=k_{2}^{2}\right)$ by integrating over the emitted electron speeds,

$$
P\left(\theta_{1}, \theta_{2}\right)=\int d k_{1} d k_{2} k_{1}^{2} k_{2}^{2} \delta\left(k_{1}-k_{2}\right) P\left(\mathbf{k}_{1}, \mathbf{k}_{2}\right),
$$

and obtain the correlated two-electron energy distribution by integrating over all angles,

$$
P\left(E_{1}, E_{2}\right)=\frac{1}{k_{1} k_{2}} \int d \Omega_{1} d \Omega_{2} P\left(\mathbf{k}_{1}, \mathbf{k}_{2}\right),
$$

where $E_{1}=k_{1}^{2} / 2$ and $E_{2}=k_{2}^{2} / 2$ are the final kinetic energies of two continuum electrons.

In order to determine the radial wave functions $\psi_{l_{1} l_{2}}^{L}\left(r_{1}, r_{2}\right)$ in (7), we adopt the FE-DVR method and numerically propagate the radial TDSE for $M=0[16,26]$,

$$
\begin{aligned}
& i \frac{\partial}{\partial t} \psi_{l_{1} l_{2}}^{L 0}\left(r_{1}, r_{2} ; t\right) \\
& \quad=\sum_{L^{\prime} l_{1}^{\prime} l_{2}^{\prime}}\left\langle l_{1}, l_{2}, L, 0|H| l_{1}^{\prime}, l_{2}^{\prime}, L^{\prime}, 0\right\rangle \psi_{l_{1}^{\prime} l_{2}^{\prime}}^{L^{\prime}}\left(r_{1}, r_{2} ; t\right),
\end{aligned}
$$

on a two-dimensional numerical grid for the radii $r_{1}$ and $r_{2}$. In order to simplify the notation we will drop the superscript $M$ throughout the remainder of this work. Details of the partialwave analysis and angular-momentum-coupling constraints are given in Appendix A.

We apply the DVR method based on the Gauss-Lobatto quadrature rule. This method is known as an efficient procedure for obtaining highly accurate results with relatively small numbers of radial grid points. Subdivision of the numerical intervals for the radial coordinates $r_{1}$ and $r_{2}$ into smaller "finite" elements translates the Hamiltonian (2) into a large sparse matrix that can be efficiently diagonalized. The FE-DVR method is well established for solving the radial two-electron TDSE and details for its implementation can be found in the literature $[16,21,22,24,26]$.

We solve (11) by numerical wave-function propagation on the $r_{1}, r_{2}$ numerical grid, repeatedly applying the split timeevolution operator [27] over equally spaced small time steps $\Delta t$,

$$
\begin{aligned}
\psi_{l_{1} l_{2}}^{L}(t+\Delta t)= & e^{-i \hat{H}_{0}(\Delta t / 2)} e^{-i \hat{V}_{\text {int }} \Delta t} e^{-i \hat{H}_{0}(\Delta t / 2)} \psi_{l_{1} l_{2}}^{L}(t) \\
& +O\left(\Delta t^{3}\right) .
\end{aligned}
$$

In order to propagate (11) in time we employ the ArnoldiLanczos method [28,29], adopting the software package EXPOKIT to perform the numerical matrix exponentiations [30]. At each numerical grid point $\left(r_{1}, r_{2}\right)$ the Hamiltonian $H_{0}$ and the interaction potential $V_{\text {int }}$ are represented as $N \times N$ matrices, $\hat{H}_{0}$ and $\hat{V}_{\text {int }}$, respectively, in the channel indices and must be diagonalized. $N$ is the total number of channels, and each channel is defined by the set of quantum numbers $\left(L, l_{1}, l_{2}\right)$.

Since the FE-DVR scheme is based on the Gauss-Lobatto quadrature rule [31], it requires the integrand to be a polynomial function for highly accurate results. However, part of our numerical effort consists in calculating the matrix elements $\left\langle\psi_{l_{1}^{\prime} l_{2}^{\prime}}^{L^{\prime}}\left(r_{1}, r_{2}\right)\left|1 /\left(\left|\mathbf{r}_{1}-\mathbf{r}_{2}\right|\right)\right| \psi_{l_{1} l_{2}}^{L}\left(r_{1}, r_{2}\right)\right\rangle$ of the electronic interaction for which the Gauss-Lobatto scheme is not appropriate. To diagonalize the potential matrix without 
loss of accuracy, we instead calculate this integral by solving Poisson's equation, following the work of McCurdy et al. [21].

\section{RESULTS AND DISCUSSION}

We submitted our new FE-DVR code to extensive tests in order to assess the accuracy of our numerical results relative to the numerical effort (in terms of computing time and random access memory) and published data. For example, our FEDVR calculations reproduce the measured [32] energies for the $1^{1} S$ ground state and the two lowest $L=0$ excited states of $\mathrm{He}$ to at least four significant digits at moderate numerical effort. Appendix B contains details of the numerical tests we performed in order to determine the convergence of energies and JADs with regard to numerical and physical parameters, such as the number of angular momenta included in (7), the size and distribution of mesh points in the $\left(r_{1}, r_{2}\right)$ numerical grid, and the time interval for which the TDSE is propagated.

All calculations in this section are carried out using a numerical grid with radial distances $0 \leqslant r_{1} \leqslant r_{1}^{\max }=277$ and $0 \leqslant r_{2} \leqslant r_{2}^{\max }=277$, including 151 FEs and 4 grid points in each FE, in combination with the Poison-equation method (cf. Sec. II and Appendix B). This grid will be referred to as "Grid 3" throughout this work, while the labels "Grid 1" and "Grid 2" are reserved for smaller grids used for numerical test in Appendix B. All joint energy and angular distributions in this section are evaluated by propagating the field-free TDSE for the time $\tau_{\max }=40$ past the end of the XUV pulse. In agreement with Zhang et al. [16] we find this time to be long enough to yield converged distributions.

\section{A. Multiphoton double ionization in a single XUV pulse}

Depending on the number of XUV photons involved, $N_{\mathrm{XUV}}$, and photon energy, $\omega_{\mathrm{XUV}}$, DI of helium in intense XUV pulses proceeds by either sequential DI or nonsequential DI. For sequential DI the two electrons independently absorb one or several photons in order to sequentially overcome the first ionization potential, $I_{p_{1}}=24.6 \mathrm{eV}$, and second ionization potential, $I_{p_{2}}=54.4 \mathrm{eV}$. In this section we discuss joint energy and angle distributions of the emitted electrons for DI of helium induced by the absorption of either one 90-eV, two $45-\mathrm{eV}$, or three $30-\mathrm{eV}$ photons. For all three cases the total energy transferred to the atom is $90 \mathrm{eV}$ (Fig. 2), and the electrons leave the atom with a combined excess energy of $E_{\text {exc }}=90 \mathrm{eV}-I_{p_{1}}-I_{p_{2}}=E_{1}+E_{2} \approx 11 \mathrm{eV}$. While DI is predominantly sequential for the absorption of three $30-\mathrm{eV}$ photons, the absorption of two $45-\mathrm{eV}$ or one $90-\mathrm{eV}$ photon can only proceed through nonsequential DI.

For all numerical results in this subsection (Sec. III A) we used the angular momentum limits $\left(L_{\max }, l_{1}^{\max }, l_{2}^{\max }\right)=(3,3,3)$ for one-photon DI and two-photon DI and $\left(L_{\max }, l_{1}^{\max }, l_{2}^{\max }\right)=$ $(5,5,5)$ for three-photon DI in the expansion (7), unless specified otherwise.

\section{Energy distributions}

Figure 3 shows joint photoelectron energy distributions for the DI of helium by one, two, and three photons, with photon energies $\hbar \omega_{\mathrm{XUV}}=90,45$, and $30 \mathrm{eV}$, respectively. The photon energies in each case are the central energy in the spectrum

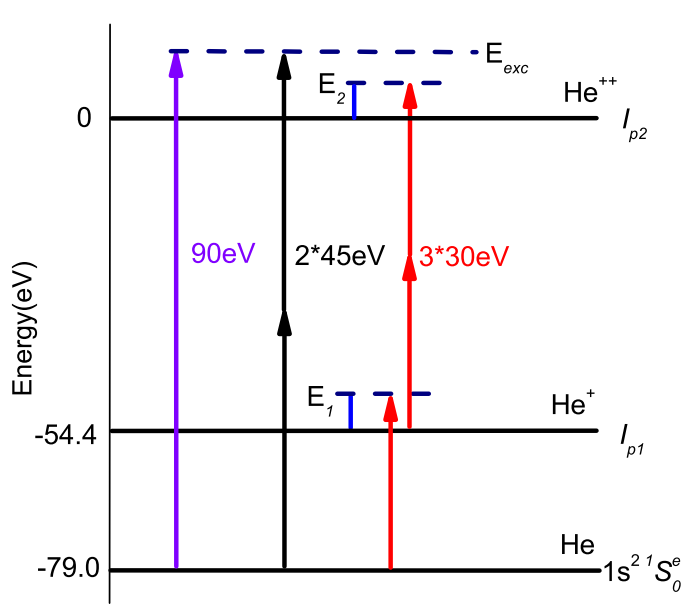

FIG. 2. (Color online) Energetics for nonsequential double ionization of helium by two $45-\mathrm{eV}$ or one $90-\mathrm{eV}$ photons and sequential double ionization by three $30-\mathrm{eV}$ photons. $I_{p_{1}}$ and $I_{p_{2}}$ designate the first and second ionization threshold, respectively. For the examples considered in this section, the combined excess energy of the emitted electrons is $E_{\mathrm{exc}}=E_{1}+E_{2}=11 \mathrm{eV}$.

of an XUV pulse with peak intensity $I_{0}=10^{14} \mathrm{~W} / \mathrm{cm}^{2}$ and pulse duration 0.5 fs [full width at half maximum (FWHM) in intensity], corresponding to the spectral width (FWHM in spectral intensity) $\hbar \Delta \omega_{\mathrm{XUV}}=3.64 \mathrm{eV}$.

As expected, the energy distributions in Figs. 3(a)-3(c) show dominant DI yields at $11 \mathrm{eV}$ excess energy. Corresponding normalized DI yields, integrated over the XUV-pulse spectral profile,

$$
P(\epsilon)=\int_{0}^{\infty} d E_{1} P\left(E_{1}, \frac{1-\epsilon}{\epsilon} E_{1}\right),
$$

are given in Fig. 3(d) as a function of the energy sharing parameter $\epsilon=E_{1} /\left(E_{1}+E_{2}\right)$ [cf. Eq. (10)]. For the spectrally broad sub-fs XUV pulses considered in this work and for nonsequential DI by one and two photons, the DI yields are distributed over a broad range of energy sharings with $E_{\text {exc }} \approx 11 \mathrm{eV}$. For nonsequential DI by a single $90-\mathrm{eV}$ photon, the DI yield is almost independent of the energy sharing between the photoelectrons [Figs. 3(a) and 3(d)], a fact that is well established in the literature, both theoretically and experimentally, and also shown for a slightly higher XUV photon energy (99 eV) in Ref. [33]. Nonsequential DI by two $45-\mathrm{eV}$ photons slightly favors asymmetric energy sharing [Figs. 3(b) and 3(d)]. Our joint energy distributions in Fig. 3(b) qualitatively resemble, but are less asymmetrical than, the distributions calculated by Guan et al. [11] for a slightly higher photon energy (48 eV).

In contrast, sequential DI by three $30-\mathrm{eV}$ photons operates by one-photon emission of the first electron with kinetic energy $E_{1} \approx \hbar \omega_{\mathrm{XUV}}-I_{p_{1}}=5.4 \mathrm{eV}$, followed by two-photon emission of the second electron, leading to the energy release $E_{2} \approx 2 \hbar \omega_{\mathrm{XUV}}-I_{p_{2}}=5.6 \mathrm{eV}$. Accordingly, the largest yield in Fig. 3(c) occurs near $E_{1} \approx E_{2}=5.5 \mathrm{eV}$. The small energy difference of $E_{2}-E_{1}=0.2 \mathrm{eV}$ is much less than $\hbar \Delta \omega_{\mathrm{XUV}}$ and thus cannot be resolved in this graph within the spectral width of the XUV pulse. 

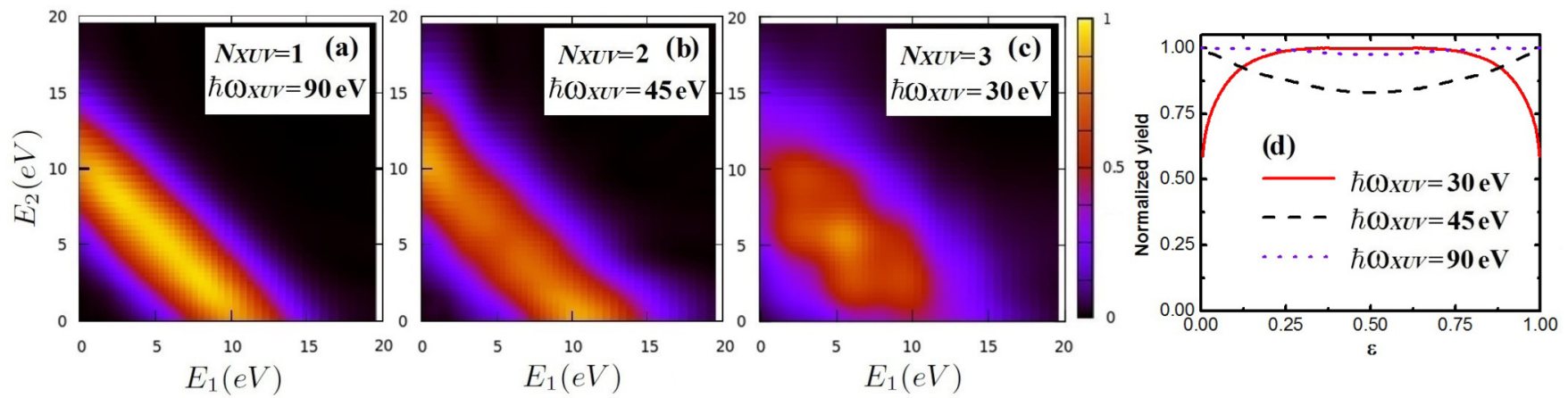

FIG. 3. (Color online) Normalized joint photoelectron energy distributions for the double ionization of helium by (a) one 90 -eV, (b) two 45-eV, and (c) three $30-\mathrm{eV}$ photons of $10^{14} \mathrm{~W} / \mathrm{cm}^{2}$ peak intensity 1-fs-long XUV pulses. (d) Corresponding normalized double-ionization yields, integrated over the XUV-pulse spectral profile, as a function of the energy sharing $\epsilon$ between the photoelectrons.

\section{Angular distributions}

In this subsection we focus on XUV DI of helium at equal energy sharing where correlation effects are expected to be strongest [34] and analyze angular distributions for the three cases in Fig. 2. Except for the XUV photon energies, we use the same laser and numerical parameters as for the energy distributions in Fig. 3. As for the preceding subsection, we separately discuss one, two, and three XUV-photon DI.

Our calculated JAD for DI by one XUV photon at equal energy sharing is shown in Fig. 4(a) for 90-eV XUV photon energy. It is dominated by four peaks on the $\theta_{1}+\theta_{2}=360^{\circ}$ axis that correspond to symmetric emission (cf. Fig. 1). It excludes back-to-back, conic, and side-by-side emission, in agreement with the selection rules B2 and F discussed by Maulbetsch and Briggs [34] and Briggs and Schmidt [20]. The absence of back-to-back emission agrees with the selection rule $\mathrm{B} 2$ which challenges the intuitive expectation that strong electronic correlation during nonsequential DI enforces backto-back emission. However, since this would violate parity conservation, emission occurs instead with an angle of $130^{\circ}$ between the emission directions of the electrons, rather than $180^{\circ}$. Side-by-side emission is forbidden because of the electronic Coulomb repulsion, and is also excluded by selection rule $\mathrm{B} 2$.

Figure 4(b) compares our calculated conditional angular distributions for single-photon DI for XUV photon energies of 90 and $99 \mathrm{eV}$ with the experimental data of Bräuning et al. [3], taken at $99 \mathrm{eV}$ (20 eV excess energy). In this polar plot the emission direction of one electron is held fixed at $\theta_{1}=60^{\circ}$. For this figure only we added results for $99 \mathrm{eV}$ in order to facilitate the comparison with the experimental data. The experimental distribution (black dots with error bars) is normalized to the absolute total DI cross section $(8.76 \mathrm{~kb})$ of Samson et al. [4]. Our calculated angular distributions for 90 and $99 \mathrm{eV}$ are very similar, however, the magnitude of the calculated $90-\mathrm{eV}$ distribution is scaled by the factor 0.55 relative to our calculated $99-\mathrm{eV}$ distribution. Our $99-\mathrm{eV}$ distribution is adjusted to the measured distribution at $\theta_{2}=330^{\circ}$ and agrees well with the experimental distribution.

A comparison of conditional angular distributions for $\theta_{1}=$ $-76^{\circ}$ and $\hbar \omega_{\mathrm{XUV}}=99 \mathrm{eV}$ is shown in Fig. 4(c). We adjusted our calculated angular distribution to the distribution measured by Schwarzkopf et al. [35] at $\theta_{2}=45^{\circ}$. The green dasheddotted line shows our result, the solid black lines are convergent
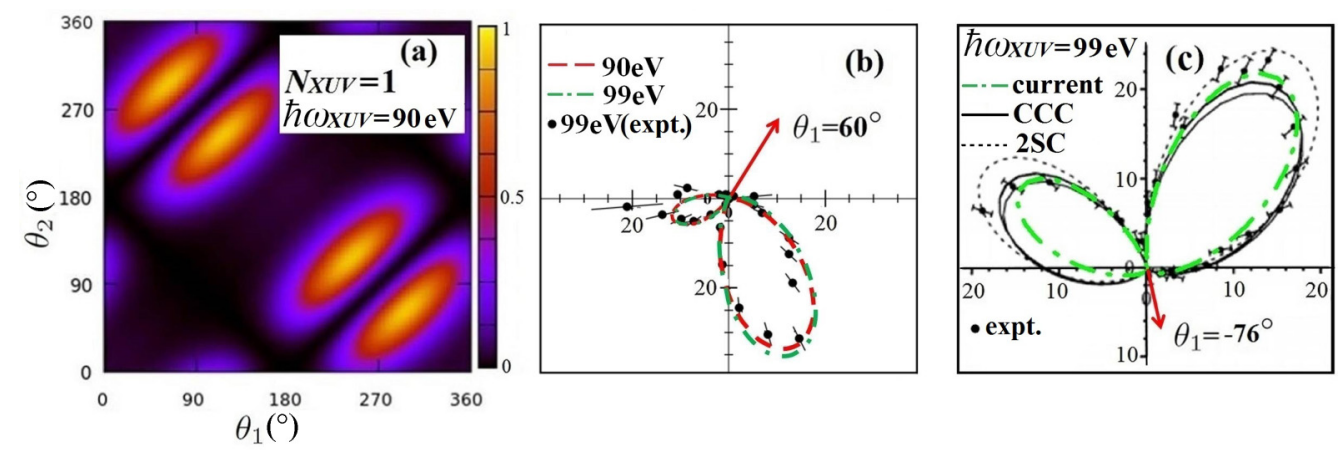

FIG. 4. (Color online) (a) Calculated normalized joint angular distribution for the double ionization of helium by one $\hbar \omega_{\mathrm{XuV}}=90-\mathrm{eV}$ XUV photon at equal energy sharing. The XUV pulse has a peak intensity of $10^{14} \mathrm{~W} / \mathrm{cm}^{2}$ and a pulse lengths of $1 \mathrm{fs}$. (b) Conditional angular distribution for $\theta_{1}=60^{\circ}$. The red dashed line shows the calculated angular distribution for $\hbar \omega_{\mathrm{XUV}}=90 \mathrm{eV}$ and is scaled by the factor 0.55 relative to the distribution calculated for $\hbar \omega_{\mathrm{XUV}}=99 \mathrm{eV}$ (green dash-dotted line). The black dots with error bars show the distribution measured by Bräuning et al. [3] for $\hbar \omega_{\mathrm{XUV}}=99 \mathrm{eV}$ in units of $\mathrm{b} \mathrm{eV}^{-1} \mathrm{sr}^{-2}$. Adapted from [3]. (c) Conditional angular distribution for $\theta_{1}=-76^{\circ}$ and $\hbar \omega_{\mathrm{XUV}}=99 \mathrm{eV}$. The green dashed-dotted line shows our result, the solid black lines are convergent close-coupling calculations for different gauges by Kheifets and Bray [36], and the black dotted line shows a calculation with screened final-state Coulomb wave functions by Pont and Shakeshaft [37]. The black dots with error bars show the measured relative cross sections of Schwarzkopf et al. [35]. Adapted from [36]. 

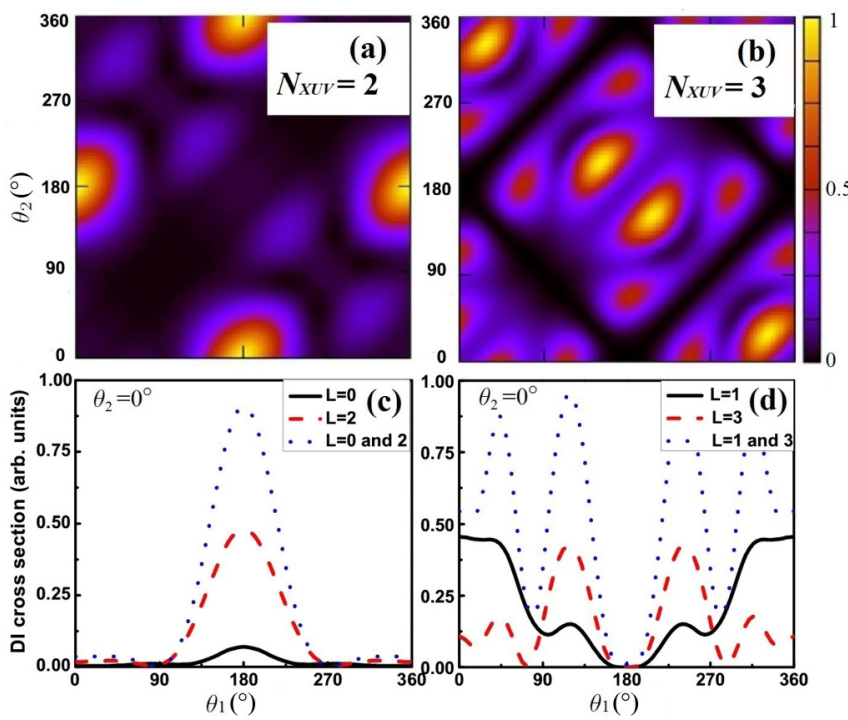

FIG. 5. (Color online) Calculated normalized (a), (b) joint angular distributions and (c), (d) conditional distributions for $\theta_{2}=0^{\circ}$ for the double ionization of helium at equal energy sharing by (a), (c) two $45-\mathrm{eV}$ photons and (b), (d) three $30-\mathrm{eV}$ photons in XUV pulses with a peak intensity of $10^{14} \mathrm{~W} / \mathrm{cm}^{2}$ and a pulse lengths of $1 \mathrm{fs}$ (see text).

close-coupling calculations (CCC) for different gauges by Kheifets and Bray [36], and the black dotted line shows a calculation with screened final-state Coulomb wave functions by Pont and Shakeshaft [37]. The three gauges used in the CCC calculation yield similar distributions, with almost indistinguishable results for the velocity and acceleration gauges.

Our calculated angle-differential yield for equal-energysharing DI of helium as a result of the absorption of two 45-eV photons is shown in Fig. 5(a). It has four distinct peaks that correspond to back-to-back emission. Thus, features prohibited for single-photon sequential DI (back-to-back and conic emission) are dominant for two-photon DI, where the preferred emission type is back to back. The same conclusions can be drawn from the theoretical equal energy sharing JADs calculated by Zhang et al. [16] for photon energies between 42 and $85 \mathrm{eV}$.

The JAD for DI by three $30-\mathrm{eV}$ photons in Fig. 5(b) is similar to the single-photon JAD in Fig. 4(a) in the sense that the same forbidden emission patterns are present. In either case, back-to-back, conic, and side-by-side emission are prohibited, as mandated by selection rules [34]. For one- and three-photon DI the JADs contain the same dominant nodal lines. We calculated the three-photon JAD by propagating for the time $\tau_{\max }=100$ past the end of XUV pulse in order to minimize unphysical side-by-side emission. For this propagation time, the contribution from side-by-side emission relative to the total DI yield for three-photon DI, integrated over a $10^{\circ}$-wide stripe ( $5^{\circ}$ on each side), centered about $\theta_{1}=\theta_{2}$, is below $1.3 \%$. Calculating the three-photon JAD for the same propagation time, $\tau_{\max }=40$, we used for the one- and two-photon JADs in Figs. 4(a) and 5(a), we found a small contribution of side-by-side emission for one- and two-photon DI, comparable to the almost converged result in Fig. 11(c) (cf. Appendix B).
Variation of $\tau_{\max }$, i.e., of the total propagation time, allows us to disentangle effects that tend to influence the JAD in opposite ways (not shown). Since three $30-\mathrm{eV}$-photon DI is (predominantly) sequential in nature, electronic correlation is not required to efficiently release both electrons. The photoelectrons thus tend to individually follow the electric force they experience in the linearly polarized XUV pulse. Furthermore, since back-to-back emission would violate parity conservation they initially tend to move in the same sense along the polarization direction. However, for equal energy sharing the effect of electron repulsion accumulates as time goes on and prevents the detection of side-by-side emission. As electron repulsion becomes increasingly influential, the initial "side-by-side electronic density" is pushed away from the $\theta_{1}=\theta_{2}$ line and merges with the nearby peaks on the symmetric emission line $\left(\theta_{2}=360^{\circ}-\theta_{1}\right)$ in Fig. 5(b) (cf. disappearance of the side-by-side contribution in Fig. 11 of Appendix B for increasing $\tau_{\max }$ ).

Normalized DI yields for the emission of one electron along the XUV polarization directions $\left(\theta_{2}=0^{\circ}\right)$ corresponding to the JADs in Figs. 5(a) and 5(b) are given in Figs. 5(c) and $5(d)$, respectively, where, in addition, contributions to the DI yields from individual allowed angular momenta $L$ of the twoelectron state are shown. These contributions are obtained by restricting the sum over $L$ in (7) to $L=0$ or 2 in Fig. 5(c) and to $L=1$ or 3 in Fig. 5(d). The shape of the angular distributions in Fig. 5 depends on the interference of the two contributing partial waves. Our two-photon angular distribution in Fig. 5(c) is dominated by $D$ waves $(L=2)$ and is in fair agreement with the CCC calculations of Kheifets and Ivanov [9] and in good agreement with the time-dependent close-coupling calculations of Colgan and Pinzola [38].

In general, successively increasing the number of absorbed XUV photons leads to JADs with alternating forbidden and dominant back-to-back and conic emission. This finding is consistent with the selection rules for equal energy sharing derived by Maulbetsch et al. [34,39] and is retained in the simplified, reduced-dimensionality model of Ni et al. [15] who constrained the center of mass of the two electrons to move on a straight line along the XUV polarization direction.

\section{Total cross sections for one and two XUV photon coplanar emission}

We calculated the total cross sections $\sigma_{\text {tot }}^{N_{\text {Xuv }}}$ for coplanar DI based on (10) by integrating over the energies $E_{1}$ and $E_{2}$ of the emitted electrons, using Eq. (11) of Ref. [16]. For DI induced by the absorption of a single $90-\mathrm{eV}$ photon we find $\sigma_{\text {tot }}^{1}=7.40 \times 10^{-21} \mathrm{~cm}^{2}$, in good agreement with the value of $7.53 \times 10^{-21} \mathrm{~cm}^{2}$ measured by Samson et al. [4]. For DI upon absorption of two 45-eV photons, we obtain $\sigma_{\text {tot }}^{2}=8.9 \times$ $10^{-53} \mathrm{~cm}^{4} \mathrm{~s}$, consistent with the cross section $\left(8.5 \times 10^{-53}\right.$ $\mathrm{cm}^{4} \mathrm{~s}$ ) calculated by Guan et al. [11].

\section{B. XUV double ionization in the presence of an IR laser pulse}

The presence of a comparatively weak IR pulse during the DI of helium in an intense XUV pulse can strongly modify the energy and angular distribution of photoelectrons. In this section we discuss and quantify effects of an assisting IR laser electric field on the XUV-DI process. The XUV and IR pulse 

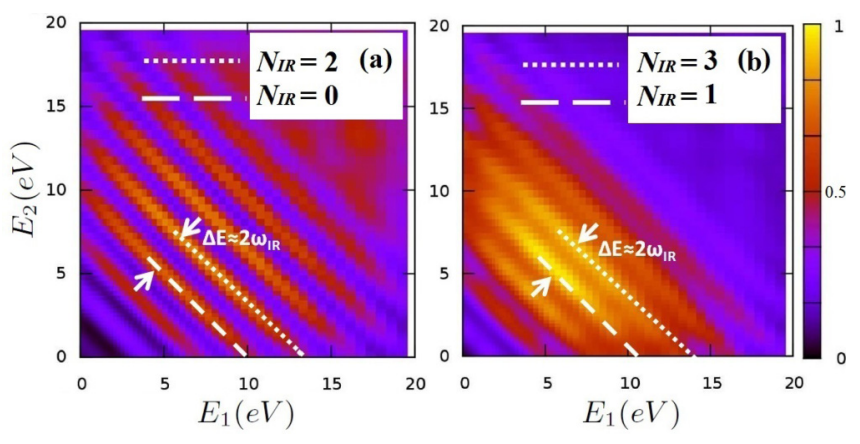

FIG. 6. (Color online) Normalized joint photoelectron energy distributions for IR-laser-assisted single-photon double ionization of helium at equal energy sharing in a 10-cycle XUV pulse of peak intensity $10^{14} \mathrm{~W} / \mathrm{cm}^{2}$, central photon energy $\hbar \omega_{\mathrm{XUV}}=89 \mathrm{eV}$, and pulse length $0.46 \mathrm{fs}$. The assisting single-cycle laser pulse has a peak intensity of $3 \times 10^{12} \mathrm{~W} / \mathrm{cm}^{2}$, photon energy of $\hbar \omega_{\mathrm{IR}}=1.61 \mathrm{eV}$, and a pulse length of $2.6 \mathrm{fs}$. (a) Even effective numbers of IR photons. (b) Odd effective numbers of IR photons.

are assumed to have overlapping cosine-squared temporal profiles, as given in (5), with identical phases $\left(\varphi_{\mathrm{XUV}}=\varphi_{\mathrm{IR}}=0^{\circ}\right)$. The XUV pulse has a peak intensity of $I_{\mathrm{XUV}}=10^{14} \mathrm{~W} / \mathrm{cm}^{2}$, the central photon energy $\hbar \omega_{\mathrm{XUV}}=89 \mathrm{eV}$, and a pulse length (full width at half intensity) of $10 \mathrm{XUV}$ cycles or $0.46 \mathrm{fs}$. The IR parameters are $I_{\mathrm{IR}}=3 \times 10^{12} \mathrm{~W} / \mathrm{cm}^{2}$, and $\hbar \omega_{\mathrm{IR}}=1.61$ $\mathrm{eV}$. At this intensity the IR pulse by itself would not ionize the atom [40]. We will discuss energy and angular distributions for assisting IR pulses with pulse lengths of 1 and 4 IR cycles or 2.6 and 10.3 fs, respectively. Unless otherwise specified, all calculations in this Sec. III B are carried out with angular momentum limits $\left(L_{\max }, l_{1}^{\max }, l_{2}^{\max }\right)=(5,5,5)$ in the expansion (7).

\section{Energy distributions}

Figure 6 shows the joint energy distributions for laserassisted single XUV photon DI for an IR pulse length of $2.6 \mathrm{fs}$. Figures 6(a) and 6(b) display joint distributions where the effective number of absorbed minus emitted IR photons is even and odd, respectively. We separated the contributions from even and odd IR photon numbers in order to clearly resolve the sideband pattern $[41,42]$ in the joint energy distribution. Each stripe in the pattern complies with energy conservation, representing a certain effective number $N_{\text {IR }}$ of absorbed minus emitted IR photons.

In each graph, neighboring stripes are separated by the energy of two IR photons $(\sim 3.2 \mathrm{eV})$, as indicated by dashed and dotted lines. Regardless of the photon number, the largest DI yield occurs at equal energy sharing. The most intense stripes in Fig. 6(a) occur at the total energies $E_{1}+E_{2} \approx 10$, 13.2, and $16.4 \mathrm{eV}$, corresponding to the absorption of one XUV photon and, effectively, 0, 2, and 4 IR photons. The largest DI yields in Fig. 6(b) are located at $E_{1}+E_{2} \approx 11.5 \mathrm{eV}$, corresponding to the absorption of (effectively) one XUV photon and one IR photon.

\section{Angular distributions for even and odd IR photon numbers}

We analyze JADs for the special case of both emitted electrons having the same final kinetic energy. As in the previous subsection, we separately consider even and odd effective photon numbers for clarity. Figures $7(a), 7(b)$, and 7(e) and Figs. 7(c), 7(d), and 7(f) show our numerical results for laser-assisted XUV-photon DI of helium for odd and even total photon numbers, respectively. We obtain these angular distributions by integrating joint energy distributions across all sidebands for equal energy sharing. Note that for odd total photon numbers in Figs. 7(a), 7(b), and 7(e) we include one 89-eV XUV plus any even number of IR photons. Likewise, for even total photon numbers in Figs. 7(c), 7(d), and 7(f), we include one XUV plus any odd number of IR photons.

Figure 7(f) shows results for laser-assisted two-photon DI, including two 45-eV XUV and any even number of IR photons. We obtain the laser-assisted single XUV-photon JADs by including combined excess energies of the two electrons up to $E_{\mathrm{exc}}^{\max }=3.0 \approx 82 \mathrm{eV}$, corresponding to $N_{\mathrm{IR}} \leqslant 45$. The IR pulse lengths are 2.6 and 10.3 fs in Figs. 7(a) and 7(c) and Figs. 7(b) and $7(d)$, respectively, corresponding to one and four IR cycles. Figures 7(e) and 7(f) compare the JADs for symmetric emission by one and two XUV photons, respectively.

The nodal lines in the JADs for single- and three XUVphoton DI of helium without laser assistance in Figs. 4(a) and 5(b) resist the addition of any even effective number of IR photons in Figs. 7(a) and 7(b). Combinations of photoelectron emission directions that are forbidden in the absence of an IR laser pulse thus remain forbidden upon addition of the laser pulse, as long as the total number of photons remains odd. Likewise, for even total photon numbers, JADs without [Fig. 5(a)] and with an assisting laser pulse [Figs. 7(c) and 7(d)] are similar and dominated by back-to-back emission. For both, even and odd photon numbers, the assistance of the IR pulse adds structure to the JAD, along lines with $\theta_{1}+\theta_{2}=$ const and most obviously for symmetric emission.

In comparison with laser-free XUV DI in Figs. 4(a) and 5, the laser-assisted yields in Fig. 7 show weak but enhanced (not observable) side-by-side emission, indicating a more pronounced influence of electronic correlation during the laser-assisted DI process. Increasing the IR pulse length from 2.6 to 10.3 fs leads to a redistribution of yields in the JADs. For odd total photon numbers, the four dominant symmetric emission peaks along $\theta_{1}+\theta_{2}=360^{\circ}$ in Fig. 7(a) recede as the IR pulse length is increased to $10.3 \mathrm{fs}$ in Fig. 7(b) and the dominant yield becomes focused in eight peaks along lines $\theta_{1}+\theta_{2}=(360 \pm 90)^{\circ}$, consistent with the transfer of discrete amounts of IR-photon momenta. For even total photon numbers, the increase of the IR pulse length from $2.6 \mathrm{fs}$ in Fig. 7(c) to 10.3 fs in Fig. 7(d) preserves the dominant back-toback emission along the laser- and XUV-polarization direction. As for odd photon numbers, the more diffuse appearance of the JADs for the longer IR pulse can be understood as the result of a prolonged momentum exchange with the IR-laser electric field during the photoemission process.

Figures 7(e) and 7(f) include cuts of the JADs in Figs. 7(a)7(d) and 5(a) along the symmetric-emission line given by $\theta_{1}+\theta_{2}=360^{\circ}$. Since convergence in the propagation time is slowest for side-by-side emission, the DI yield at $\theta_{1}=180^{\circ}$ in Figs. 7(e) and 7(f) allows us to quantify the overall degree of convergence achieved in the numerical calculation. For a fully converged calculation this yield has to vanish. In general, our JADs are closest to convergence for laser-free 

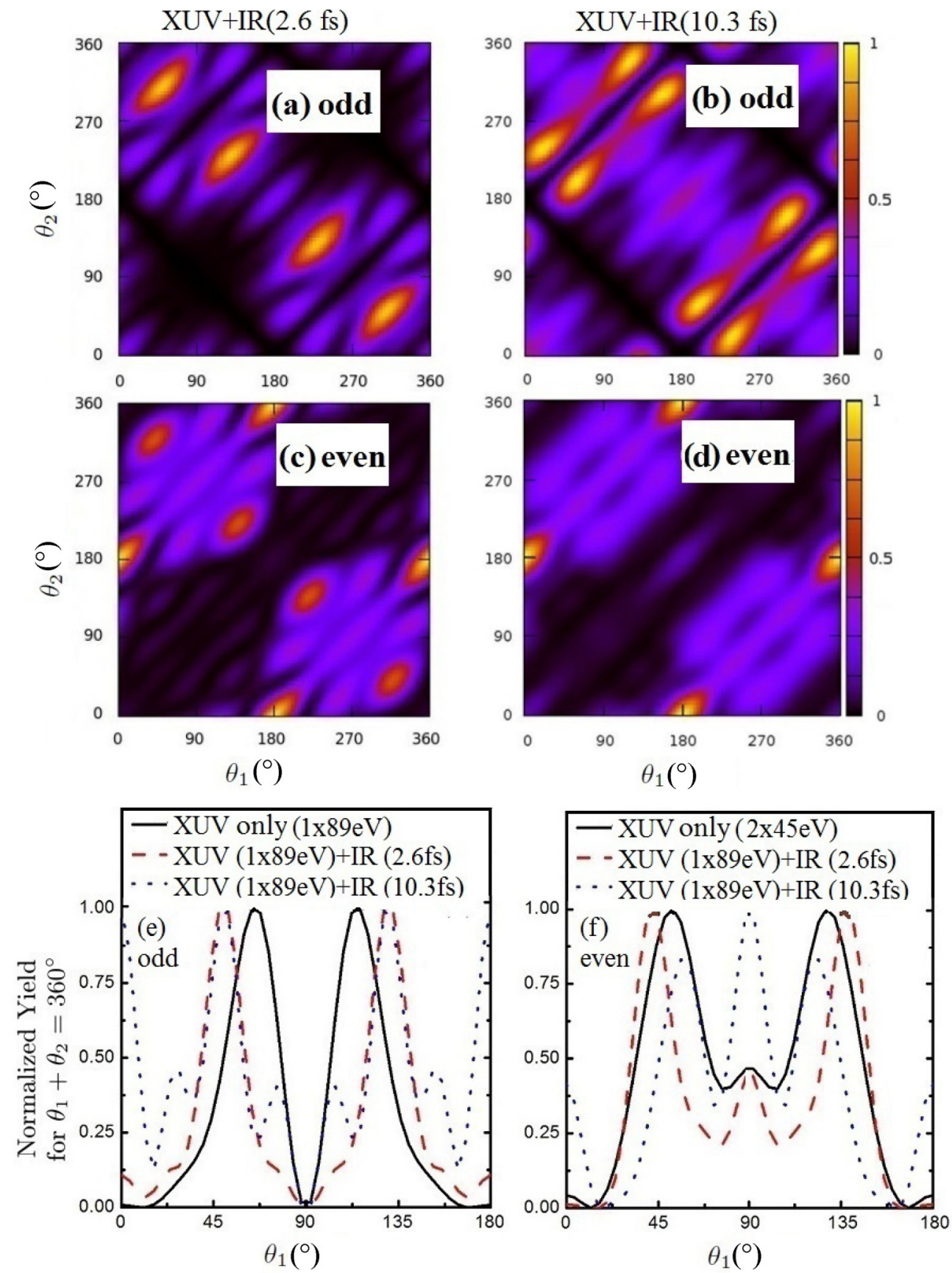

FIG. 7. (Color online) Normalized joint angular distributions for IR-laser-assisted double ionization of helium at equal energy sharing in a 10 -cycle XUV pulse of peak intensity $10^{14} \mathrm{~W} / \mathrm{cm}^{2}$ and central photon energies of $\hbar \omega_{\mathrm{XUV}}=89$ and $45 \mathrm{eV}$. The assisting IR pulse has a peak intensity of $3 \times 10^{12} \mathrm{~W} / \mathrm{cm}^{2}$, central photon energy $\hbar \omega_{\mathrm{IR}}=1.61 \mathrm{eV}$, and pulse lengths (a), (c) $2.6 \mathrm{fs}$ and (b), (d) $10.3 \mathrm{fs}$. (a)-(d) Laser-assisted single-89 eV-photon double ionization for (a), (b) odd and (c), (d) even total effective numbers of XUV and IR photons. (e) Normalized single-89 eV-photon double ionization for symmetric emission with and without assisting IR-laser pulses of pulse lengths 2.6 and 10.3 fs for odd total effective numbers of XUV and IR photons. (f) Normalized single-89-eV-photon double ionization for symmetric emission with an assisting 2.6 or 10.3 fs IR-laser pulse for even total effective numbers of XUV and IR photons, compared with IR-laser-free two-45-eV-photon double ionization. Calculation with angular momentum limits $\left(L_{\max }, l_{1}^{\max }, l_{2}^{\max }\right)=(4,4,4)$ in the expansion (7).

XUV DI and least converged for the longest pulse lengths of the assisting laser, for otherwise identical physical and numerical parameters. The deterioration of the convergence induced by the assisting laser pulse is more severe for odd photon numbers in Fig. 7(e).

\section{Angular distributions for specific effective IR photon numbers}

Figure 8 shows normalized JADs for single-XUV photon DI of helium for specific even and odd total numbers of photons within the range $-1 \leqslant N_{\mathrm{XUV}}+N_{\mathrm{IR}} \leqslant 4$. Negative (positive) IR-photon numbers $N_{\text {IR }}$ indicate that the number of emitted 

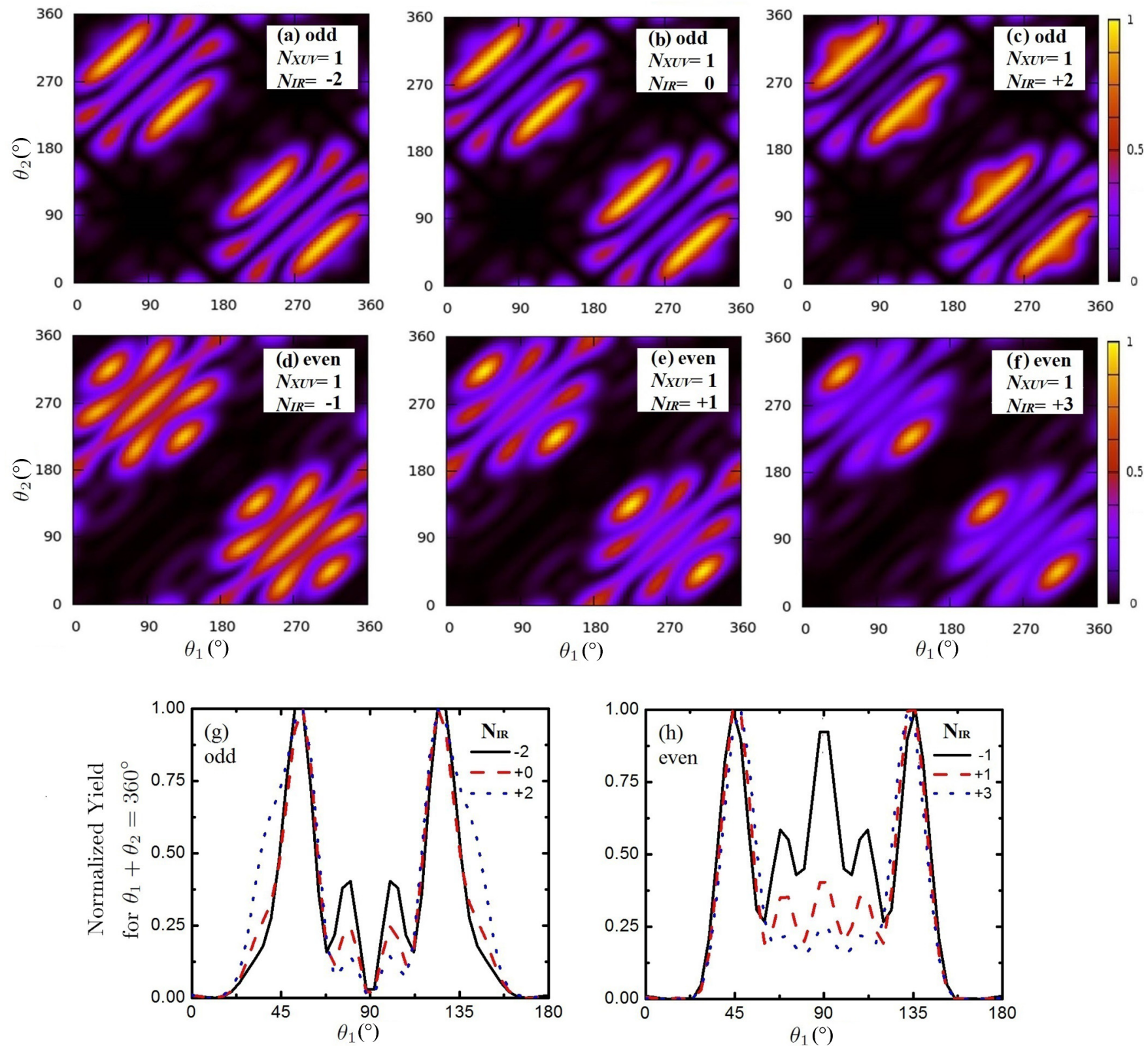

FIG. 8. (Color online) Normalized joint angular distributions for IR-laser-assisted single-XUV-photon double ionization of helium in a 10 -cycle XUV pulse of peak intensity $10^{14} \mathrm{~W} / \mathrm{cm}^{2}$ and central photon energy (a)-(f) $\hbar \omega_{\mathrm{XUV}}=90 \mathrm{eV}$. The assisting IR laser pulse has a pulse length of $2.6 \mathrm{fs}$. All other parameters are the same as for Fig. 7. (a)-(c), (g) Odd and (d)-(f), (h) even total effective numbers of photons. The effective number of absorbed IR photons is (a) $N_{\mathrm{IR}}=-2$, (b) 0 , (c) 2, (d) -1 , (e) 1 , and (f) 3 . Negative photon numbers indicate that more IR photons are emitted than absorbed. (g), (h) Normalized yields for symmetric laser-assisted XUV double ionization. (g) Odd total numbers of photons with $N_{\mathrm{IR}}=-2,0$, and 2. (g) Even total numbers of photons with $N_{\mathrm{IR}}=-1,1$, and 3 .

IR photons exceeds (is smaller than) the number of absorbed IR photons. The pulse length of the assisting IR laser pulse is 2.6 fs. All other laser, XUV-pulse, and numerical parameters are the same as in the preceding Sec. III B 2. These JADs were obtained from the energy distributions in Fig. 6 by integrating over a small energy domain near equal energy sharing given by

$$
\left|E_{1}+E_{2}-E_{\mathrm{exc}}\right|<\omega_{\mathrm{IR}}
$$

where $E_{\mathrm{exc}}=N_{\mathrm{XUV}} \omega_{\mathrm{XUV}}+N_{\mathrm{IR}} \omega_{\mathrm{IR}}-I_{p_{1}}-I_{p_{2}}$ is the total excess energy due to the (net) absorption of $N_{\mathrm{XUV}}(=1,2)$ XUV and $N_{\text {IR }}$ IR photons. As for all JADs shown in this paper, each JAD is normalized separately to its maximum yield.

The IR-photon-number specific JADs in Figs. 8(a)-8(f) resemble the distribution for unspecified odd and even photon numbers in Figs. 7(a) and 7(c). For odd photon numbers, the DI yields become more concentrated in the four prominent peaks at and near symmetric emission while all other structures lose intensity as $N_{\text {IR }}$ is increased from -2 to 2 in Figs. 8(a)-8(c). As observed for laser-free XUV emission in Fig. 4(a) and for laser-assisted emission in Figs. 7(a) and 7(b), back-to-back and conic emission remain separately forbidden for each 
number of IR photons $N_{\mathrm{IR}}$, as long as the total photon number is odd.

For even $N_{\text {IR }}$ symmetric emission is most likely. The progressive concentration of the DI yield in four peaks along the symmetric emission direction as $N_{\text {IR }}$ increases from -2 to 3 in Figs. 8(a)-8(f) is more pronounced for even than for odd total photon numbers. As for laser-free DI by absorption of two XUV photons in Fig. 5(a) back-to-back emission contributes significantly for laser-assisted XUV DI with $N_{\text {IR }}=$ -1 [Fig. 8(d)]. However, as more IR photon are effectively absorbed, the momenta transferred from the IR-laser field onto the photoelectrons progressively weaken back-to-back emission and increase the relative importance of symmetric emission in the dominant four peaks in Figs. 8(e) and 8(f). For increasing numbers of effectively absorbed IR photons, symmetric emission thus becomes more dominant for both odd and even photon numbers. For equal energy sharing of the photoelectrons, this can be regarded as a compromise between (i) the simultaneous transfer of equal amounts of momenta along the laser and XUV pulse polarization direction from the IR-laser field to the emitted electrons (promoting side-by-side emission) and (ii) electronic repulsion (prohibiting side-byside emission at sufficiently large propagation times).

The normalized DI yields for symmetric laser-assisted XUV DI for odd total photon numbers in Fig. 8(g) show a steady decrease of the DI yield near the back-to-back emission direction at $\theta_{1} \approx(90 \pm 12)^{\circ}$ as $N_{\mathrm{IR}}$ is increased from -2 to +2 . Simultaneously, the dominant symmetric emission peaks in this graph at $\theta_{1} \approx 55^{\circ}$ and $\approx 125^{\circ}$ become broader, as one would expect for larger numbers of absorbed IR photons. For the case of even total photon numbers in Fig. 8(h) symmetric back-to-back emission in direction perpendicular to the laser polarization direction $\left(\theta_{1}=90^{\circ}\right)$ becomes less likely as $N_{\text {IR }}$ is increased from -1 to +3 , in agreement with the intuitive picture of increasing momentum being transferred from the external IR electric field to the photoelectrons in direction parallel to the laser polarization.

Our discussion shows that for equal energy sharing, backto-back emission oscillates between forbidden (for odd) and dominant (for even totol photon numbers $N_{\mathrm{XUV}}+N_{\mathrm{IR}}$ ) as the number of effectively absorbed IR photon increases. This oscillation originates in dipole and parity selection rules $[7,34,39]$.

\section{SUMMARY}

We investigated the IR laser-assisted XUV DI of helium by applying the FE-DVR method for numerically solving the TDSE equation in full dimensionality. We validated our numerical results by comparison with experimental angular distributions and by assessing the convergence in the number of included angular momentum eigenstates, propagation time, and numerical grid size. Our discussion focused on equal energy sharing of the emitted electrons, where for side-by-side emission convergence in the propagation time is slowed by the Coulomb interaction of the emitted electrons and thus serves as a lower limit for the degree of convergence at different emission angles (and nonequal energy sharing). We classified the distinct back-to-back, side-by-side, conic, and symmetric double emission modes in JADs at equal energy sharing and discussed their prominence and absence for sequential and nonsequential DI upon absorption of up to three XUV photons and with and without the presence of an assisting IR laser field.

We investigated JADs separately for odd and even numbers of exchanged photons and for specific effective numbers of absorbed (and emitted) IR photons. We found that an assisting IR field retains some of the dominant features of the laser-free JADs while modifying the distribution of DI yields in compliance with known dipole and parity selection rules. These modifications become more significant as the effective number of absorbed IR photons increases, in support of the intuitive picture of momentum transfer from the IR-laser field to the photoemitted electrons in the laser-polarization direction. In particular, we found that the IR pulse promotes side-by-side and enables back-to-back emission. For even numbers of absorbed (IR plus XUV) photons, the IR pulse was found to enable back-to-back emission and conic emission. This is in agreement with the dipole and parity selection rules that forbid (allow) back-to-back and conic emission for odd (even) total effective numbers of (IR plus XUV) photons, respectively.

We plan to extend this study to nonequal energy sharing. We also intend to apply our code to systematically investigate the influence of the carrier-envelope phases of the XUV and IR pulse, the XUV-pulse length, and the delay between XUV and IR pulse have on the correlated electron dynamics during the double-ionization process.

\section{ACKNOWLEDGMENTS}

We thank to W. Cao, and Q. Liao for helpful discussions and X. Guan for advice on the FE-DVR method. This work was supported by the United States National Science Foundation (NSF) under Grant No. PHY-1068752 and the Division of Chemical Sciences, Office of Basic Energy Sciences, Office of Energy Research, and US Department of Energy. Part of this research used computational resources of (i) the National Energy Research Scientific Computing Center, which is supported by the Office of Science of the US Department of Energy under Contract No. DE-AC02-05CH11231 and (ii) the Beocat Research Cluster at Kansas State University, which is funded in part by NSF Grants No. CNS-1006860, No. EPS-1006860, and No. EPS-0919443.

\section{APPENDIX A: RADIAL TDSE AND COEFFICIENTS OF ANGULAR MOMENTUM COUPLING FUNCTIONS}

The radial TDSE (11) can be written as

$$
\begin{aligned}
i \frac{\partial}{\partial t} \psi_{l_{1} l_{2}}^{L}\left(r_{1}, r_{2} ; t\right)= & {\left[\hat{T}+\hat{V}_{c}\right] \psi_{l_{1} l_{2}}^{L}\left(r_{1}, r_{2} ; t\right) } \\
& +\sum_{L^{\prime} l_{1}^{\prime} l_{2}^{\prime}} \hat{V}_{F_{0}}\left(\lambda, \lambda^{\prime}\right) \psi_{l_{1}^{l_{1}} l_{2}^{\prime}}^{L^{\prime}}\left(r_{1}, r_{2} ; t\right) \\
& +\sum_{L^{\prime} l_{1}^{\prime} l_{2}^{\prime}} \hat{V}_{F_{12}}\left(\lambda, \lambda^{\prime}\right) \psi_{l_{1}^{\prime} l_{2}^{\prime}}^{L^{\prime}}\left(r_{1}, r_{2} ; t\right) .
\end{aligned}
$$

$\hat{T}=-(1 / 2) \partial^{2} / \partial r_{1}^{2}=-(1 / 2) \partial^{2} / \partial r_{2}^{2}$ is the radial kinetic energy operator for the two electrons, $r_{<}=\min \left\{r_{1}, r_{2}\right\}, r_{>}=$ 
$\max \left\{r_{1}, r_{2}\right\}$, and

$$
\hat{V}_{c}=-\frac{2}{r_{1}}+\frac{l_{1}\left(l_{1}+1\right)}{2 r_{1}^{2}}-\frac{2}{r_{2}}+\frac{l_{2}\left(l_{2}+1\right)}{2 r_{2}^{2}}
$$

includes the Coulomb interactions with the nucleus and the centrifugal potentials. The angular-momentum coupling is included in

$$
\hat{V}_{F_{0}}\left(\lambda, \lambda^{\prime}\right)=\sum_{l=0}^{\infty} \frac{4 \pi(-1)^{l}}{\sqrt{2 l+1}} \frac{r_{<}^{l}}{r_{>}^{l+1}} F_{0}\left(\lambda, \lambda^{\prime}\right)
$$

for the electronic interaction and

$$
\hat{V}_{F_{12}}\left(\lambda, \lambda^{\prime}\right)=\frac{4 \pi}{\sqrt{3}} E(t)\left[r_{1} F_{1}\left(\lambda, \lambda^{\prime}\right)+r_{2} F_{2}\left(\lambda, \lambda^{\prime}\right)\right]
$$

for the interaction of the external electric field $E(t)=E_{\mathrm{XUV}}+$ $E_{\mathrm{IR}}$, where the collective angular momentum quantum numbers are defined as $\lambda=\left\{L, l_{1}, l_{2}\right\}, \lambda^{\prime}=\left\{L^{\prime}, l_{1}^{\prime}, l_{2}^{\prime}\right\}$.

Following Ref. [26], the angular-momentum coupling matrix elements can be written as

$$
\begin{aligned}
F_{0}= & \sqrt{(2 l+1)^{2}\left(2 l_{1}^{\prime}+1\right)\left(2 l_{2}^{\prime}+1\right)\left(2 L^{\prime}+1\right) /(4 \pi)^{2}} \\
& \times C_{l 0 l_{1}^{\prime} 0}^{l_{1} 0} C_{l 0 l_{2}^{\prime} 0}^{l_{2} 0} C_{00 L^{\prime} 0}^{L 0} \times\left\{\begin{array}{lll}
l & l_{1}^{\prime} & l_{1} \\
l & l_{2}^{\prime} & l_{2} \\
0 & L^{\prime} & L
\end{array}\right\} \\
F_{1}= & \sqrt{9\left(2 l_{1}^{\prime}+1\right)\left(2 l_{2}^{\prime}+1\right)\left(2 L^{\prime}+1\right) /(4 \pi)^{2}} \\
& \times C_{10 l_{1}^{\prime} 0}^{l_{1} 0} C_{00 l_{2}^{\prime} 0}^{l_{2} 0} C_{10 L^{\prime} 0}^{L 0} \times\left\{\begin{array}{lll}
1 & l_{1}^{\prime} & l_{1} \\
0 & l_{2}^{\prime} & l_{2} \\
1 & L^{\prime} & L
\end{array}\right\}
\end{aligned}
$$

and

$$
\begin{aligned}
F_{2}= & \sqrt{9\left(2 l_{1}^{\prime}+1\right)\left(2 l_{2}^{\prime}+1\right)\left(2 L^{\prime}+1\right) /(4 \pi)^{2}} \\
& \times C_{00 l_{1}^{\prime} 0}^{l_{1} 0} C_{10 l_{2}^{\prime} 0}^{l_{2} 0} C_{10 L^{\prime} 0}^{L 0} \times\left\{\begin{array}{ccc}
0 & l_{1}^{\prime} & l_{1} \\
1 & l_{2}^{\prime} & l_{2} \\
1 & L & L^{\prime}
\end{array}\right\},
\end{aligned}
$$

where Clebsch-Gordan coefficients and $9 j$ symbols [43] are denoted as $C_{\ldots}^{\ldots}$ and $\{\ldots\}$, respectively.
In terms of the collective quantum numbers $\lambda$ and $\lambda^{\prime}$, (A1) can be cast in the more compact form

$$
\begin{aligned}
i \frac{\partial}{\partial t} \psi_{\lambda}\left(r_{1}, r_{2} ; t\right)= & {\left[\hat{T}+\hat{V}_{c}\right] \psi_{\lambda}\left(r_{1}, r_{2} ; t\right)+\sum_{\lambda^{\prime}}\left[\hat{V}_{F_{0}}\left(\lambda, \lambda^{\prime}\right)\right.} \\
& \left.+\hat{V}_{F_{12}}\left(\lambda, \lambda^{\prime}\right)\right] \psi_{\lambda^{\prime}}\left(r_{1}, r_{2} ; t\right)
\end{aligned}
$$

\section{APPENDIX B: NUMERICAL TESTS}

a. Convergence with respect to $l_{1}$ and $l_{2}$

As a first test, we computed the ground and two first excited $L=0$ state energies of helium. Using the imaginary time propagation method [44], we replaced the real time variable $t$ in our FE-DVR code by the imaginary time variable $\tau=i t$ and numerically propagated (12) in imaginary time. We started this propagation with an initial two-electron trial wave function for the helium ground state, given as the product of Gaussian functions,

$$
\psi_{l_{1} l_{2}}^{00}\left(r_{1}, r_{2} ; t=0\right)=A e^{-\left(r_{1}-r_{0}\right)^{2}-\left(r_{2}-r_{0}\right)^{2}}
$$

with the normalization constant $A$, total angular momentum quantum numbers $L=M=0$, and $r_{0}=10$.

To test our code, we represented the radial distances $0 \leqslant$ $r_{1} \leqslant r_{1}^{\max }=57.2$ and $0 \leqslant r_{2} \leqslant r_{2}^{\max }=57.2$ on two different numerical grids. For each radial distance, Grid 1 consists of 50 FEs with 4 grid points in each FE. Taking overlapping end points of neighboring FEs into account, this results in $50 \times(4-1)-1=149$ points for Grid 1. Similarly, Grid 2 contains 20 elements with 10 grid points in each FE, resulting in a total of 179 grid points for each radial coordinate.

We repeatedly applied (12), propagating the TDSE for $0.5 \mathrm{fs}$ with equidistant time steps for predetermined maximal values $l_{1}{ }^{\max }$ and $l_{2}{ }^{\max }$ of $l_{1}$ and $l_{2}$. We used the same value for each time step as $\mathrm{Hu}$ [26], $\Delta t=0.0041$. For $0 \leqslant l_{1} \leqslant l_{1}^{\max }=3$, $0 \leqslant l_{2} \leqslant l_{2}^{\max }=3$, and $L=0$, we found that the ground-state energy has converged with regard to the propagation time and number of time steps to at least four significant decimal digits on either Grid 1 or Grid 2.

Our energies for the $1^{1} S$ ground state of He, computed by FE-DVR imaginary-time propagation for $l_{1}{ }^{\max }=l_{2}{ }^{\max }=3$, including the four channels $\left(L, l_{1}, l_{2}\right)=(0,0,0),(0,1,1),(0,2,2)$, and $(0,3,3)$, agree to four significant digits with our result

TABLE I. $1^{1} S$ ground-state and the lowest two $L=0$ excited-state energies, $2^{1} S$ and $3^{1} S$, of helium. The correlation matrix elements (radial matrix elements of $r_{<}^{l} / r_{>}^{l+1}$, cf. Appendix A) are computed by either Gauss-Labatto integration (referred to as "Direct integration") or by solving Poisson's equation ("Poisson eq."). The FE-DVR imaginary time propagation calculations were performed for two different radial numerical grids covering $0 \leqslant r_{1} \leqslant r_{1}^{\max }=57.2$ and $0 \leqslant r_{2} \leqslant r_{2}^{\max }=57.2$ : Grid 1 uses 50 elements with 4 grid points in each element, and Grid 2 uses 20 elements with 10 grid points in each element. The three last rows show theoretical [16,45] and experimental [32] energies from the literature.

\begin{tabular}{llll}
\hline \hline Method/Source & \multicolumn{1}{c}{$1^{1} S$} & $2^{1} S$ & $3^{1} S$ \\
\hline Direct integration (Grid 1) & -2.8896 & -2.1301 & -2.1446 \\
Direct integration (Grid 2) & -2.9017 & -2.1459006 & -2.0612720 \\
Poisson eq. (Grid 1) & -2.9033635 & -2.1459706 & -2.0612710 \\
Theoretical value [16] & -2.9036690 & -2.1459740 & -2.0612720 \\
Theoretical value [45] & -2.9037244 & -2.1458986 & -2.0612017 \\
Experimental value [32] & -2.9035612 & & \\
\hline \hline
\end{tabular}



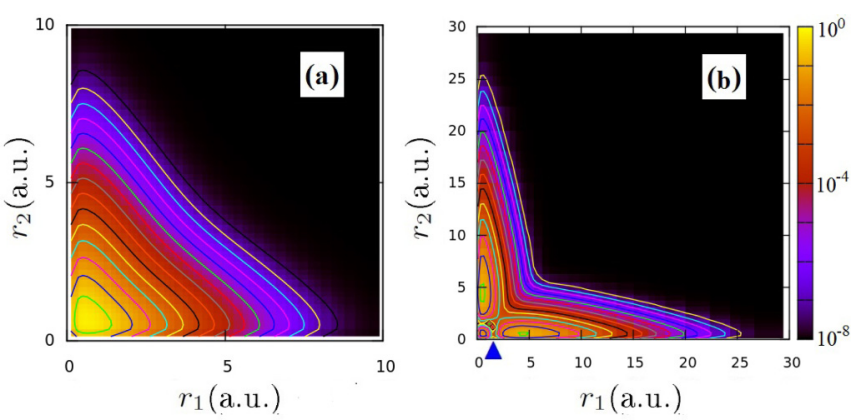

FIG. 9. (Color online) Probability densities of the (a) $1^{1} S$ ground state and (b) $2^{1} S$ first excited state of helium obtained by imaginary time propagation on a logarithmic color (gray) scale. The blue solid triangle in (b) indicates the node in $r_{1}$ direction of the first excited-state radial wave function.

for $l_{1}{ }^{\max }=l_{2}{ }^{\max }=10$, including $11 L=0$ channels. Our calculated ground-state energies also coincide to four significant digits with published experimental [32] and recently calculated theoretical [16] energies (Table I).

Following the same imaginary time-propagation procedure, while projecting the converged ground state out of the trial wave function (B1) every ten time steps, we obtain the energies of the first excited $2^{1} S$ state listed in the third column of Table I. Similarly, repeatedly projecting both the converged ground and first excited states out of (B1) every ten time steps during the numerical wave-function propagation, we arrive at the second excited zero-angular-momentum $3{ }^{1} S$ state energy in the fourth column. We obtain our most accurate values for the energies of the $2^{1} S$ state, -2.1459006 , and the $3^{1} S$ state, -2.0612720 , by applying the "Poisson-equation method" (see below). Our first and second excited $L=0$ state energies are in good agreement with the theoretical values, -2.1459706 and -2.0612710 , calculated by Zhang et al. [16] using the same FE-DVR method with parameters $l_{1}^{\max }=l_{2}^{\max }=7, r_{1}^{\max }=$ $r_{2}^{\max }=57.2$ divided into 64 elements, and with the measured energies, -2.1458986 and -2.0612017 of Martin [32], respectively. The corresponding radial probability densities,

$$
p\left(r_{1}, r_{2}\right)=\sum_{L l_{1} l_{2}}\left|\psi_{l_{1} l_{2}}^{L 0}\left(r_{1}, r_{2}\right)\right|^{2},
$$

are calculated on numerical Grid 1 by imaginary time propagation for the ground and first excited state and shown in Fig. 9. The ground-state probability density has a maximum at $r_{1}=r_{2} \approx 0.9$. The first excited state has maxima at $r_{1}=r_{2} \approx$ 0.5 and $r_{1}=r_{2} \approx 4.5$, and a node at $r_{1}=r_{2} \approx 1.7$.

\section{b. Convergence with respect to the numerical grid size}

We compared the ground-state and first-excited-state energies for imaginary time propagation calculations with different numbers of FEs and different numbers of grid points in each element. We performed these calculations for $L=0$ and $l_{1}{ }^{\max }=l_{2}{ }^{\max }=3$.

Table I includes our results for the ground- and first-excitedstate energies of helium. As listed in the first two rows, the calculation on Grid 1 results in the energies -2.8896 and -2.1301 , while using Grid 2 we obtain -2.9017 and
-2.1446 , respectively, for the $1^{1} S$ and $2{ }^{1} S$ states of helium. As expected, the numerical grid with more points (Grid 2) yields results in better agreement with the measured energies (last row).

\section{c. Comparison of the "Poisson equation" and "direct integration" methods}

We next compared results for the lowest helium energies obtained by either diagonalizing the matrices representing the electronic correlation terms $r_{<}^{l} / r_{>}^{l+1}\left[r_{<} \equiv \min \left(r_{1}, r_{2}\right)\right.$, and $\left.r_{>} \equiv \max \left(r_{1}, r_{2}\right)\right]$ in (11) and (A1) via Gauss-Lobatto integration or by solving Poisson's equation [21]. Knowing that the Poisson equation method is better suited to overcome numerical problems related to the singular $1 /\left|r_{2}-r_{1}\right|$ electronic correlation interaction, it is nevertheless worth pointing out that our ground- and excited-state energies agree better with the calculated energies of Zhang et al. [16] and the measured data by Martin [32] for calculations with the smaller numerical grid (Grid 1) in combination with Poisson's equation than for calculations carried out on the larger grid (Grid 2) in conjunction with a straightforward Gauss-Lobatto integration. Using the Poisson equation method and the smaller Grid 1 we find that the three lowest helium energies in Table I are converged to at least four significant digits and agree to at least three significant digits with published experimental [32] and recently calculated theoretical [16] energies.

The ground-state energy $(-2.90367)$ obtained by Zhang et al. [16] is in slightly better agreement with the experimental value $(-2.90356)$ than our best value in Table I (-2.903 36). This is to be expected in view of the numerically significantly more expensive FE-DVR calculation by Zhang et al. with parameters $r_{1}^{\max }=r_{2}^{\max }=57.2, l_{1}^{\max }=l_{2}^{\max }=7,64 \mathrm{FEs}$, and 8 grid points in each element. Our best numerical value for the ground-state energy in Table I agrees equally well with the result of a calculation using an explicitly correlated basis, -2.90372 , by Scrinzi et al. [45].

The third and fourth column of Table I compare first- and second-excited $L=0$ state energies from different sources. Our best values for the energies of the $2^{1} S$ state (dominated by the $1 s 2 s$ configuration), -2.1459006 , and $3{ }^{1} S$ state (1s $3 s$ dominated), -2.0612720 , are in good agreement with the theoretical values, -2.1459706 and -2.0612710 , of Zhang et al. [16], -2.1459740 and -2.0612720 of Scrinzi et al. [45], and the experimental values, -2.1458986 and -2.0612017 , of Martin et al. [32], respectively. Our values for the excited-state energies agree to five digits with the measured energies, while we reproduce the measured ground-state energy to three digits. We note that the experimental energies quoted in Table I also agree slightly better with both relativistic and nonrelativistic calculations by Alexander et al. [46] for the excited states than the ground state.

\section{d. Convergence in the number of included total angular momenta $L$}

In this subsection, we discuss the convergence of joint angular distributions with regard to the number of total angular momentum states $\psi_{l_{1} l_{2}}^{L M}$ included in (7) for the IR-laser-pulseassisted XUV DI of helium. The central frequency, intensity, and pulse length for the XUV and IR pulses are $\hbar \omega_{\mathrm{XUV}}=$ 

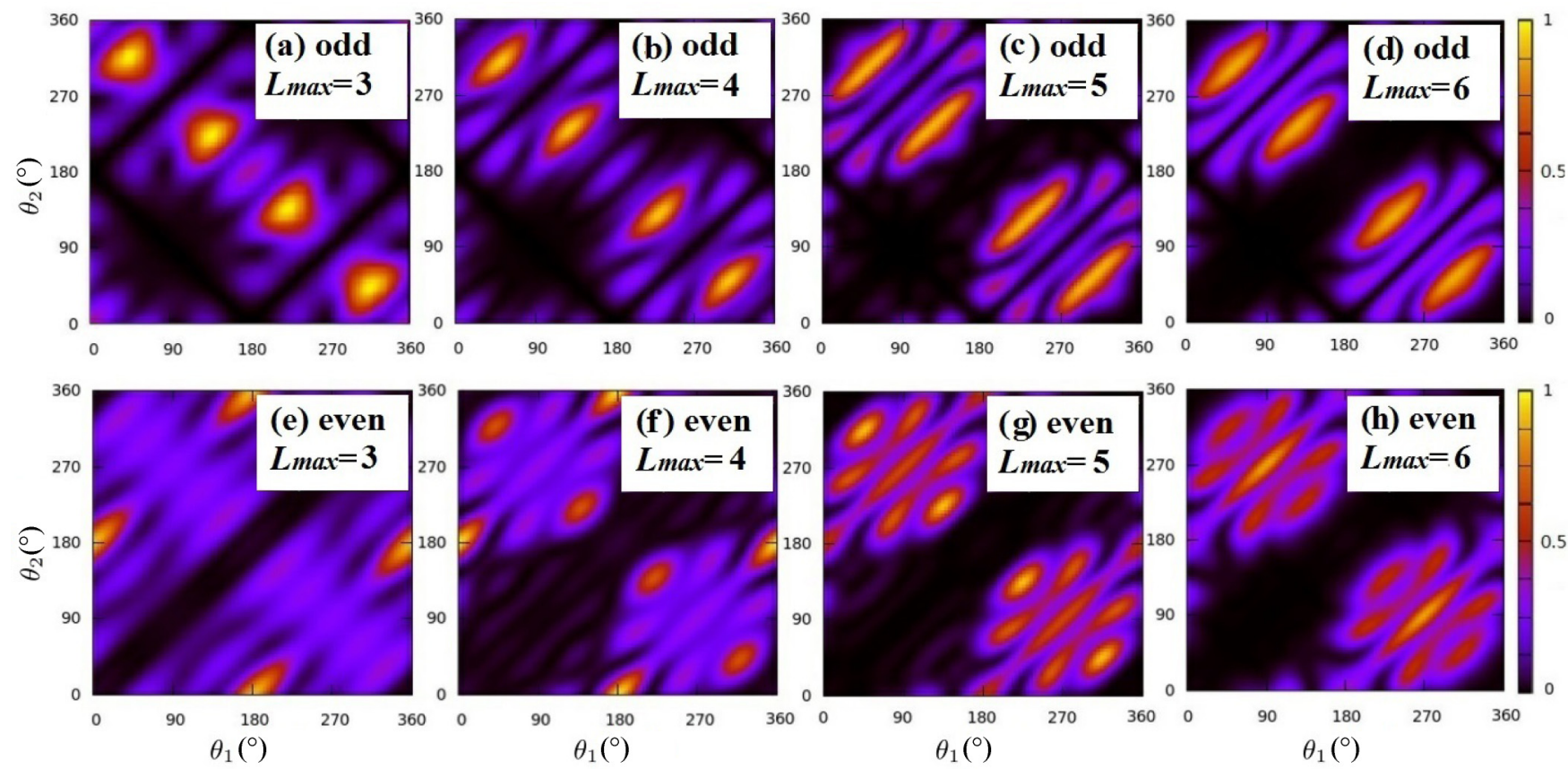

FIG. 10. (Color online) Normalized joint angular distributions for IR-laser-assisted XUV double ionization of helium at equal energy sharing for maximal angular momentum quantum numbers (a), (e) $\left(L_{\max }, l_{1}^{\max }, l_{2}^{\max }\right)=(3,3,3),(\mathrm{b}),(\mathrm{f})(4,4,4),(\mathrm{c}),(\mathrm{g})(5,5,5)$, and (d), (h) $(6,6,6)$. (a)-(d) Results for odd and (e)-(h) even photon numbers. The photon numbers correspond to one absorbed XUV plus the number of absorbed minus emitted IR photons.

$89 \mathrm{eV}, I_{\mathrm{XUV}}=10^{14} \mathrm{~W} / \mathrm{cm}^{2}, \tau_{\mathrm{XUV}}=0.47 \mathrm{fs}(10 \mathrm{XUV}$ optical cycles), and $\hbar \omega_{\mathrm{IR}}=1.61 \mathrm{eV}, I_{\mathrm{IR}}=3 \times 10^{12} \mathrm{~W} / \mathrm{cm}^{2}, \tau_{\mathrm{IR}}=$ 2.6 fs (1 IR optical cycle), respectively. We assume that the two linearly polarized pulses coincide (no time delay) with equal energy sharing of the emitted electrons $\left(E_{1}=E_{2}\right)$. We performed these calculations for the numerical Grid 3 (defined in Sec. III A), with 151 elements, 4 grid points in each FE, the first FE length (near the origin) set equal to 0.5 , and $r_{1}^{\max }=r_{2}^{\max } \approx 277$. Grid 3 is an extension of Grid 1 with an extra 101 elements.

The graphs in Fig. 10 show JADs for IR-laser-assisted XUV DI obtained from separate calculations for maximal angular momentum quantum numbers, $\left(L_{\max }, l_{1}^{\max }, l_{2}^{\max }\right)=(3,3,3)$, $(4,4,4),(5,5,5)$, and $(6,6,6)$, corresponding to 23,42 , 69, and 106 channels, respectively. All angular distributions are snapshots taken 20 atomic time units after the end of the cosine-squared-shaped IR laser pulse (5). The graphs in the top (bottom) row show angular distributions for odd (even) total photon numbers.

The JADS in Fig. 10 converge with increasing $L_{\max }$ and the not observable, low probability for side-by-side emission becomes negligible. While the intensity distributions for $L_{\max }=4,5$, and 6 show small discrepancies, their main structures are the same, and the JADs appear to have converged at $L_{\max }=5$ with regard to their main characteristics. For this reason we use $\left(L_{\max }, l_{1}^{\max }, l_{2}^{\max }\right)=(5,5,5)$, corresponding to 69 partial-wave channels, for all subsequent numerical results discussed in the work, unless specified otherwise.

\section{e. Convergence in the propagation time}

Figure 11 shows normalized JADs for laser-assisted XUV DI of helium for equal energy sharing of the emitted electrons. The calculations are performed on Grid 3, using
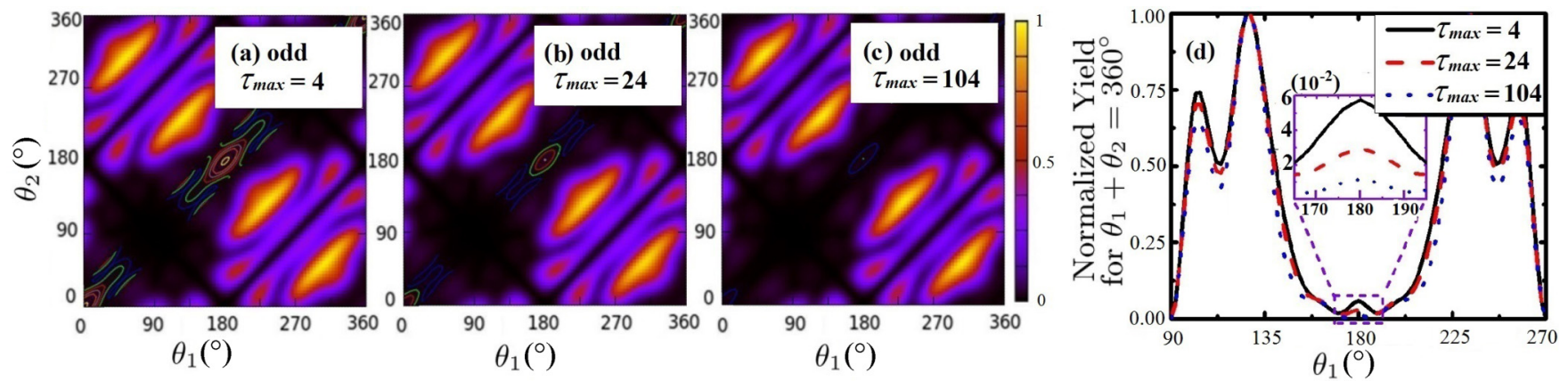

FIG. 11. (Color online) Normalized joint angular distributions for IR-laser-assisted XUV-double ionization of helium at equal energy sharing for different propagation times $\tau_{\max }$ past the end of the IR pulse. (a) $\tau_{\max }=4$; (b) $\tau_{\max }=24$; (c) $\tau_{\max }=104$. (d) Normalized double-ionization yields at $\theta_{1}+\theta_{2}=360^{\circ}$. As the propagation time is increased, the side-by-side-emission yield at $\theta_{1}=\theta_{2}=180^{\circ}$ disappears, as physically expected. 
the Poisson-equation method for the electron-correlationinteraction matrix elements and the angular-momentum limits $\left(L_{\max }, l_{1}^{\max }, l_{2}^{\max }\right)=(3,3,3)$. The three graphs display snapshots of JADs calculated according to (9) and taken at different propagation times $\tau_{\max }$ past the end of IR pulse.

The JAD for $\tau_{\max }=4$ in Fig. 11(a) differs from the JAD for $\tau_{\max }=24$ in Fig. 11(b), mainly with regard to the smaller not observable contribution of side-by-side emission (central dot in each graph). This contribution is physically prohibited at large distances from the residual helium nucleus due to electronic repulsion. For $\tau_{\max }=104$ the JAD has converged [Fig. 11(c)], is indistinguishable from Fig. 11(b), except for side-by-side emission, and no longer displays side-by-side emission. Indicative for the contribution of side-by-side emission for finite $\tau_{\max }$ and degree of convergence is the absolute value of the JAD at $\theta_{1}=\theta_{2}=180^{\circ}$. These absolute values are not shown in the normalized graphs of Figs. 11(a)-11(c). They decrease from 0.059 to 0.029 to 0.01 for $\tau_{\max }=4,24$, and 104 in Figs. 11(a)-11(c), respectively. We performed a similar convergence test for even photon numbers (not shown) and found much faster convergence in $\tau_{\max }$ than for odd photon numbers.

We display in Fig. 11(d) the normalized angular yields obtained from the JADs in Figs. 11(a)-11(c) on the diagonal defined by $\theta_{2}=360^{\circ}-\theta_{1}$. This graph thus highlights the four dominant peaks in Figs. 11(a)-11(c) and side-by-side emission with $\theta_{1}=\theta_{2}=0$ or $180^{\circ}$, where convergence in $\tau_{\max }$ is most difficult to achieve.

For an alternative quantitative comparison of the convergence in $\tau_{\max }$, we calculated relative integrated side-by-side yields obtained by integrating the JADs in Figs. 11(a)-11(c) over a narrow diagonal stripe centered at $\theta_{2}=\theta_{1}$. The width of this stripe is taken as $10^{\circ}$, i.e., $\left|\theta_{1}-\theta_{2}\right| \leqslant 5^{\circ}$. The relative integrated side-by-side yield drops from $0.24 \%$ at $\tau_{\max }=4$ to $0.13 \%$ at $\tau_{\max }=24$, and to $0.05 \%$ at $\tau_{\max }=104$, while the side-by-side-emission-peak heights decrease from 0.06 to 0.03 , and finally to 0.01 , respectively. Thus, as physically demanded, side-by-side emission disappears for sufficiently long propagation times, while for relative emission angles of the two photoelectrons outside the side-by-side range converged JADs are readily obtained at the end of the IR pulse. In particular, the nodal structure in Fig. 11 for non-side-by-side emission is not affected by increasing $\tau_{\max }$, as expected from the selection rules discussed by Maulbetsch and Briggs [34].

\section{f. Computing time and memory requirements}

The numerical results in Figs. 10 and 11 were obtained using the open message parallel processing protocol (OpenMP) on a $3.40-\mathrm{GHz}$ Intel i7-2600 central processing unit (CPU) with exclusive use of 24 cores and access to $30 \mathrm{~GB}$ of random access memory (RAM). The CPU time for the results shown in any of the graphs in Figs. 10 and 11 did not exceed $4700 \mathrm{~h}$ (about $200 \mathrm{~h}$ wall-clock time).

For the angular-momentum limits $\left(L_{\max }, l_{1}^{\max }, l_{2}^{\max }\right)=$ $(5,5,5)$, corresponding to 69 channels, the RAM requirement in these calculations is of the order $4 \times 8 \times N^{2} n_{\text {grid }}^{2} / 1024^{3}$ GB. The factor 4 stands for the two large coupling matrices $\hat{V}_{F_{0}}$ and $\hat{V}_{F_{12}}$ and two corresponding transformation matrices used for their diagonalization (cf. Appendix A). The factor 8 is related to our use of double-precision variables. $N$ is the number of channels defined in Sec. II above and $n_{\text {grid }}$ is the number of grid points in each of the two radial dimensions.
[1] O. Schwarzkopf, B. Krässig, J. Elmiger, and V. Schmidt, Phys. Rev. Lett. 70, 3008 (1993).

[2] O. Schwarzkopf and V. Schmidt, J. Phys. B: At. Mol. Opt. Phys. 29, 1877 (1996).

[3] H. Bräuning, R. Dörner, C. L. Cocke, M. H. Prior, B. Krässig, A. S. Kheifets, I. Bray, A. Bräuning-Demian, K. Carnes, S. Dreuil, V. Mergel, P. Richard, J. Ullrich, and H. SchmidtBöking, J. Phys. B 31, 5149 (1998).

[4] J. A. R. Samson, W. C. Stolte, Z. X. He, J. N. Cutler, Y. Lu, and R. J. Bartlett, Phys. Rev. A 57, 1906 (1998).

[5] H. Hasegawa, E. J. Takahashi, Y. Nabekawa, K. L. Ishikawa, and K. Midorikawa, Phys. Rev. A 71, 023407 (2005).

[6] A. Rudenko, L. Foucar, M. Kurka, Th. Ergler, K. U. Kühnel, Y. H. Jiang, A. Voitkiv, B. Najjari, A. Kheifets, S. Lüdemann, T. Havermeier, M. Smolarski, S. Schössler, K. Cole, M. Schöffler, R. Dörner, S. Düsterer, W. Li, B. Keitel, R. Treusch, M. Gensch, C. D. Schröter, R. Moshammer, and J. Ullrich, Phys. Rev. Lett. 101, 073003 (2008).

[7] F. Maulbetsch and J. S. Briggs, J. Phys. B: At. Mol. Opt. Phys. 26, 1679 (1993).

[8] J. S. Parker, L. R. Moore, K. J. Meharg, D. Dundas, and K. T. Taylor, J. Phys. B: At. Mol. Opt. Phys. 34, L69 (2001).

[9] A. S. Kheifets and I. A. Ivanov, J. Phys. B: At. Mol. Opt. Phys. 39, 1731 (2006).
[10] A. Y. Istomin, E. A. Pronin, N. L. Manakov, S. I. Marmo, and A. F. Starace, Phys. Rev. Lett. 97, 123002 (2006).

[11] X. Guan, K. Bartschat, and B. I. Schneider, Phys. Rev. A 77, 043421 (2008).

[12] A. Palacios, D. A. Horner, T. N. Rescigno, and C. W. McCurdy, J. Phys. B 43, 194003 (2010).

[13] S. A. Sørngård, S. Askeland, R. Nepstad, and M. Førre, Phys. Rev. A 83, 033414 (2011).

[14] H. Bachau, Phys. Rev. A 83, 033403 (2011).

[15] H. Ni, S. H. Chen, C. Ruiz, and A. Becker, J. Phys. B: At. Mol. Opt. Phys. 44, 175601 (2011).

[16] Z. Zhang, L. Y. Peng, M. H. Xu, A. F. Starace, T. Morishita, and Q. Gong, Phys. Rev. A 84, 043409 (2011).

[17] Q. Liao, Y. Zhou, C. Huang, and P. Lu, New J. Phys. 14, 013001 (2012).

[18] S. X. Hu, Phys. Rev. Lett. 111, 123003 (2013).

[19] M. Brauner, J. S. Briggs, and H. Klar, J. Phys. B: At. Mol. Opt. Phys. 22, 2265 (1989).

[20] J. S. Briggs and V. Schmidt, J. Phys. B: At. Mol. Opt. Phys. 33, R1 (2000).

[21] C. W. McCurdy, M. Baertschy, and T. N. Rescigno, J. Phys. B: At. Mol. Opt. Phys. 37, R137 (2004).

[22] B. I. Schneider, L. A. Collins, and S. X. Hu, Phys. Rev. E 73, 036708 (2006). 
[23] L. Avaldi and A. Huetz, J. Phys. B: At. Mol. Opt. Phys. 38, S861 (2005).

[24] W. C. Jiang, L. Y. Peng, W. H. Xiong, and Q. Gong, Phys. Rev. A 88, 023410 (2013).

[25] A. R. Barnett, in Computational Atomic Physics, edited by K. Bartschat (Springer, Heidelberg, New York, 1996), Chap. 9, p. 181.

[26] S. X. Hu, Phys. Rev. E 81, 056705 (2010).

[27] T. Niederhausen and U. Thumm, Phys. Rev. A 77, 013407 (2008).

[28] T. J. Park and J. C. Light, J. Chem. Phys. 85, 5870 (1986).

[29] X. Guan, O. Zatsarinny, K. Bartschat, B. I. Schneider, J. Feist, and C. J. Noble, Phys. Rev. A 76, 053411 (2007).

[30] R. B. Sidje, ACM Trans. Math. Softw. 24, 130 (1998).

[31] M. Abramowitz and I. Stegun, Handbook of Mathematical Functions (Dover, Mineola, NY, 1972), Chap. 25, p. 888.

[32] W. C. Martin, J. Phys. Chem. Ref. Data 2, 257 (1973).

[33] J. Colgan, M. S. Pinzola, and F. Robicheaux, J. Phys. B: At. Mol. Opt. Phys. 34, L457 (2001).

[34] F. Maulbetsch and J. S. Briggs, J. Phys. B: At. Mol. Opt. Phys. 28, 551 (1995).

[35] O. Schwarzkopf, B. Krässig, and V. Schmidt, J. Phys. (Paris) 3, C6 169 (1993); O. Schwarzkopf, Ph.D. thesis, University of Freiburg, Germany, 1995.
[36] A. S. Kheifets and I. Bray, J. Phys. B: At. Mol. Opt. Phys. 31, L447 (1998).

[37] M. Pont and R. Shakeshaft, Phys. Rev. A 51, R2676 (1995).

[38] J. Colgan and M. S. Pindzola, Phys. Rev. Lett. 88, 173002 (2002).

[39] F. Maulbetsch, M. Pont, J. S. Briggs, and R. Shakeshaft, J. Phys. B: At. Mol. Opt. Phys. 28, L341 (1995).

[40] B. Walker, B. Sheehy, L. F. DiMauro, P. Agostini, K. J. Schafer, and K. C. Kulander, Phys. Rev. Lett. 73, 1227 (1994).

[41] C.-H. Zhang and U. Thumm, Phys. Rev. A 80, 032902 (2009).

[42] M. Meyer, P. Radcliffe, T. Tschentscher, J. T. Costello, A. L. Cavalieri, I. Grguras, A. R. Maier, R. Kienberger, J. Bozek, C. Bostedt, S. Schorb, R. Coffee, M. Messerschmidt, C. Roedig, E. Sistrunk, L. F. Di Mauro, G. Doumy, K. Ueda, S. Wada, S. Düsterer, A. K. Kazansky, and N. M. Kabachnik, Phys. Rev. Lett. 108, 063007 (2012).

[43] W. R. Johnson, Atomic Structure Theory: Lectures on Atomic Physics (Springer, Berlin, Heidelberg, New York, 2007), Chap. 4, p. 126.

[44] F. He, C. Ruiz, A. Becker, and U. Thumm, J. Phys. B: At. Mol. Opt. Phys. 44, 211001 (2011).

[45] A. Scrinzi and B. Piraux, Phys. Rev. A 58, 1310 (1998).

[46] S. A. Alexander, S. Datta, and R. L. Coldwell, Phys. Rev. A 81, 032519 (2010). 